\title{
Full Motion and Flow Field Recovery From Echo Doppler Data
}

\author{
Muthuvel Arigovindan, Michael Sühling, Christian Jansen, Patrick Hunziker, and Michael Unser*
}

\begin{abstract}
We present a new computational method for reconstructing a vector velocity field from scattered, pulsed-wave ultrasound Doppler data. The main difficulty is that the Doppler measurements are incomplete, for they do only capture the velocity component along the beam direction. We thus propose to combine measurements from different beam directions. However, this is not yet sufficient to make the problem well posed because 1) the angle between the directions is typically small and 2) the data is noisy and nonuniformly sampled. We propose to solve this reconstruction problem in the continuous domain using regularization. The reconstruction is formulated as the minimizer of a cost that is a weighted sum of two terms: 1) the sum of squared difference between the Doppler data and the projected velocities 2) a quadratic regularization functional that imposes some smoothness on the velocity field. We express our solution for this minimization problem in a $B$-spline basis, obtaining a sparse system of equations that can be solved efficiently. Using synthetic phantom data, we demonstrate the significance of tuning the regularization according to the a priori knowledge about the physical property of the motion. Next, we validate our method using real phantom data for which the ground truth is known. We then present reconstruction results obtained from clinical data that originate from 1) blood flow in carotid bifurcation and 2) cardiac wall motion.
\end{abstract}

Index Terms-Color Doppler imaging, color flow imaging, echocardiography, nonuniform sampling, projected sampling, pulsed wave Doppler, regularized reconstruction, shift-invariant spaces, tissue Doppler imaging, ultrasound Doppler, variational reconstruction, vector field reconstruction, velocity field reconstruction.

\section{INTRODUCTION}

$\mathbf{P}$ ULSED-WAVE ultrasound Doppler (PWD) imaging is an effective tool for the monitoring of vascular and cardiac function [1]-[3]. The imaging system sends a periodic pulse train along a set of scan lines and measures the backscattered signal. By analyzing the Doppler frequency-shift in the received signal, the system retrieves a set of axial velocity estimates; these are the projected components of the true three-dimensional (3-D) velocity along the direction of the ultrasound beam.

Manuscript received February 17, 2006; revised August 15, 2006. This work was supported in part by the Swiss Heart Foundation and the Swiss National Science Foundation under Grant 200020-101821. Asterisk indicates corresponding author.

M. Arigovindan and M. Sühling are with Ecole Polytechnique Fédérale de Lausanne (EPFL), Biomedical Imaging Group, CH-1015 Lausanne, Switzerland.

C. Jansen and P. Hunziker are with the Kantonsspital Basel, CH-4031 Basel, Switzerland (e-mail: christian.jansen@unibas,ch; phunziker@uhbs.ch).

*M. Unser is with Ecole Polytechnique Fédérale de Lausanne (EPFL), Biomedical Imaging Group, CH-1015 Lausanne, Switzerland (e-mail: michael.unser@epfl.ch).

Digital Object Identifier 10.1109/TMI.2006.884201
These axial components are resampled on a regular grid and presented in a color coded form that is known as the color flow image. See [1] for a comprehensive treatment of the various aspects of such systems. In the context of clinical echocardiography, PWD imaging is better known as color Doppler imaging when used to assess blood flow, and tissue Doppler imaging, when applied to tissue motion.

The color flow image sequence contains the instantaneous velocity information, but it is incomplete; in particular, the system is blind to the motion that is orthogonal to the beam. Nevertheless, such a partial velocity field has been used for the determination of quantitative parameters such as flow volume [4]-[6]. Further, qualitative and to some extent quantitative motion analysis obtained from this kind of data was found to be clinically useful in several instances [7], [2], [8]-[10], [3]. It can, therefore, be expected that the availability of full vector field should lead to a better quantitative analysis.

An alternative to the Doppler methods is to use computational means to recover a full velocity field from a temporal sequence of $B$-mode intensity images. This is typically done using optical flow algorithms; a comparative evaluation of such techniques in the context of echocardiography can be found in [11]. A recent enhancement involves the use of a local affine model that is well adapted to the motion of the heart [12]. One can also think of improving the performance of optical flow techniques by combining it with Doppler data to improve the reconstruction quality of this local affine method [13]. Even though these $B$-mode motion analysis techniques hold promises for clinical practice, they have not superseded Doppler imaging which is generally believed to be more reliable and applicable to a wider range of situations. In that respect, the down-sides of optical flow techniques are:

1) They do not work at all for estimating blood flow because there is no visible structure on which to lock.

2) These methods are all based on the assumption that a material point gives rise to the same intensity value in successive frames. It is still not entirely clear to which extent this assumption is valid in ultrasound imaging because of the presence of multiple scatters that induce speckle which is not necessarily correlated with movement [14], [15].

3) There is some lack of temporal resolution because optical flow computations are based on frame-to-frame differences. This is in contrast with Doppler measurements for which the equivalent averaging time (proportional to the inverse of the pulse repetition rate) is significantly less. Doppler imaging would therefore come out as the method of choice for assessing motion if it were not for its basic limitation of only providing partial velocity measurements; i.e., projections along the direction of the beam. 


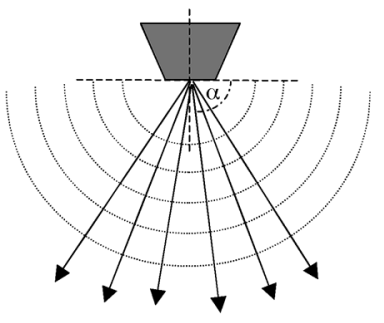

(a)

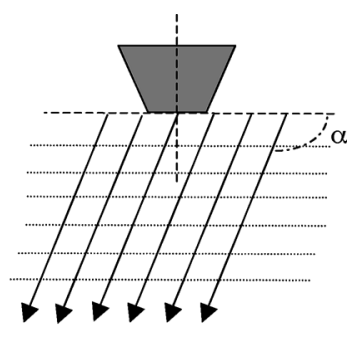

(b)
Fig. 1. Scan-line schemes for 2-D acquisition. (a) Cone-beam or sector-scan acquisition. (b) Parallel-beam acquisition.

In this paper, we propose a new approach for reconstructing the true velocity field from the PWD data only. It is distinct and complementary to the optical flow techniques in the sense that it is also intended to work for blood flow estimation. We formulate the task as the problem of finding a continuous vector function that is the minimizer of a suitable criterion. Specifically, we consider a quadratic cost functional that is a weighted sum of a data term and a physically inspired smoothness functional (regularizer). The regularizer enforces some coupling between $x$ and $y$ velocity components. We demonstrate that the partial nature of the data makes this coupling crucial.

We search for the continuous solution in a shift-invariant space, since it allows, by choosing the step size, a trade-off between computational complexity and reconstruction accuracy. We choose a $B$-spline basis for the shift-invariant space, since it gives the best trade-off, as justified in Section III-E. The $B$-spline solution is conveniently expressed in the form of a sparse system of linear equations, thanks to the exact computation of derivatives of the regularization functional [16].

Using a synthetic phantom, we demonstrate the superiority of our coupled regularizer over the conventional regularizers. We also validate our method using a real phantom experiment. Finally, we present reconstruction results for blood flow in the carotid bifurcation and cardiac wall motion along with some quantitative validation.

\section{RECONSTRUCTION PROBLEM}

In this section, we give a brief description of the form of a typical PWD data set. Then, based on a simple motion model, we describe the indeterminacy of the Doppler data.

\section{A. Form of the Data}

A Doppler imaging system measures axial velocities at some selected locations in the cross-sectional plane under consideration. The selected locations $\left\{\mathbf{x}_{i}\right\}$ are typically on a noncartesian grid. These nonuniform measurements are scan-converted (resampled in a regular grid) and then displayed in a color-coded form. A typical two-dimensional (2-D) data set will contain the set of sampling locations $\left\{\mathbf{x}_{i}\right\}$, the corresponding beam directions $\left\{\mathbf{d}_{i}\right\}$, and the Doppler measurements $\left\{m_{i}\right\}$ satisfying $m_{i}=\mathbf{d}_{i}^{T} \mathbf{v}\left(\mathbf{x}_{i}\right)$, where $\mathbf{v}(\mathbf{x})$ is the true velocity field. Note that the sampling locations $\left\{\mathbf{x}_{i}\right\}$ are nonuniform, especially when there are multiple views.

Fig. 1 gives the schematic of two commonly used sampling geometries. The solid lines with arrows represent the scan lines (ultrasound beam), whereas the dotted lines represent the sampling grid along the scan lines. In the cone-beam or sector-beam scheme, the beams are launched from a single point along a series of regularly spaced angles. In the parallel-beam acquisition, beams are launched with a fixed angle from a set of points spaced regularly along a line. A typical parallel-beam probe has the capability of steering the beam angle, which means that different Doppler images can be acquired without moving the probe (multiple acquisitions).

In this paper, we consider two types of data sets: 1) samples of multiple acquisitions of the same cross-sectional plane obtained from a cone-beam probe placed at different locations (sample locations and beam directions are transformed into a common reference plane); and 2) multiple acquisitions from a parallelbeam probe obtained by beam steering.

\section{B. Deficiency of Doppler Data}

Now we will demonstrate the deficiency of Doppler data with the help of a toy example. Specifically, let us consider a plane rotating with an angular velocity $\omega$ about the center $\left(x_{c}, y_{c}\right)$; its velocity field is given by

$$
\begin{aligned}
& u(x, y)=-\omega\left(y-y_{c}\right) \\
& v(x, y)=\omega\left(x-x_{c}\right)
\end{aligned}
$$

where $u$ and $v$ are the $x$ and $y$ components of the velocity field. Let the measurement device be a cone beam probe located at the origin of the coordinate system. The probe measures the samples of the following function:

$$
v_{d}(x, y)=\left[d_{x}(x, y) d_{y}(x, y)\right]\left[\begin{array}{l}
u(x, y) \\
v(x, y)
\end{array}\right] .
$$

Here $\left[d_{x}(x, y) d_{y}(x, y)\right]^{T}$ is the direction of the beam from the probe, whose components, in the present geometry, are given by

$$
d_{x}(x, y)=\frac{x}{\sqrt{x^{2}+y^{2}}} \quad d_{y}(x, y)=\frac{y}{\sqrt{x^{2}+y^{2}}}
$$

Then $v_{d}(x, y)$ reads

$$
v_{d}(x, y)=\frac{x \omega y_{c}-y \omega x_{c}}{\sqrt{x^{2}+y^{2}}}
$$

It is clear from the above equation that we cannot recover the motion parameters $\left\{\omega, x_{c}, y_{c}\right\}$ individually from the samples of $v_{d}(x, y)$, no matter how many. We can only recover the products $\omega x_{c}$ and $\omega y_{c}$. In other words, any rotating plane with an angular velocity, $\omega_{1}$, and a center of rotation, $\left(x_{1}, y_{1}\right)$, is a candidate solution, provided $\omega_{1} x_{1}=\omega x_{c}$ and $\omega_{1} y_{1}=\omega y_{c}$. Hence, there is no reconstruction method that can recover the motion uniquely.

We can resolve this ambiguity if we have the value of one of the components ( $u$ or $v$ ) at any point. In fact, the minimal measurement set required to determine all the three parameters is the following 1) samples of the function $v_{d}(x, y)$ at two points that do not lie on the same scan line 2) a sample of one of the components. Since it is not practical to obtain a measurement of 
a single velocity component, the only feasible way to resolve the ambiguity of Doppler data is to combine multiple acquisitions, which is the strategy adopted in this work.

\section{PROPOSED METHOD}

Let $\mathbf{v}(\mathbf{x})=[u(x, y) v(x, y)]^{T}$ be the velocity field. Our aim is to recover $\mathbf{v}(\mathbf{x})$ from the given Doppler measurement set $\left\{\mathbf{x}_{i}, \mathbf{d}_{i}, m_{i}\right\}$. We search for the solution in the space of uniform $B$-splines; in other words, we restrict the velocity field to be of the following form:

$$
\begin{aligned}
& u(x, y)=\sum_{k=0}^{N_{g}-1} \sum_{l=0}^{N_{g}-1} c_{k, l}^{u} \beta^{n}\left(\frac{x}{a}-k\right) \beta^{n}\left(\frac{y}{a}-l\right) \\
& v(x, y)=\sum_{k=0}^{N_{g}-1} \sum_{l=0}^{N_{g}-1} c_{k, l}^{v} \beta^{n}\left(\frac{x}{a}-k\right) \beta^{n}\left(\frac{y}{a}-l\right) .
\end{aligned}
$$

Here, $\beta^{n}$ is the $B$-spline of degree $n$ and $a$ is the step size that controls the accuracy. $N_{g}$ is the grid size. The idea is to formulate the reconstruction as the minimizer of a quadratic functional. Consequently, the expansion coefficients $c_{k, l}$ are expressed as a solution of a linear system of equation. We study three forms of the quadratic functional.

\section{A. Least Squares Method (LS)}

The least squares $B$-spline solution is the minimizer of the following quadratic cost functional:

$$
J_{\mathrm{LS}}(\mathbf{v})=\sum_{i=1}^{N}\left(\mathbf{d}_{i}^{T} \mathbf{v}\left(\mathbf{x}_{i}\right)-m_{i}\right)^{2}
$$

Let $\mathbf{c}_{u}=\left[\cdots c_{k, l}^{u} \cdots\right]^{T}, \mathbf{c}_{v}=\left[\cdots c_{k, l}^{v} \cdots\right]^{T}$, and $\mathbf{c}=\left[\mathbf{c}_{u}^{T} \mathbf{c}_{v}^{T}\right]^{T}$. Now, the task is to express the above functional in terms of $\mathbf{c}$. Let $\mathbf{t}_{u}=\left[\begin{array}{lll}\cdots & u\left(\mathbf{x}_{i}\right) & \cdots\end{array}\right]^{T}$ and $\mathbf{t}_{v}=\left[\begin{array}{lll}\cdots & v\left(\mathbf{x}_{i}\right) & \cdots\end{array}\right]^{T}$. The vectors $\mathbf{t}_{u}$ and $\mathbf{t}_{v}$ are computed as

$$
\begin{aligned}
& \mathbf{t}_{u}=\mathbf{S}_{s} \mathbf{c}_{u} \\
& \mathbf{t}_{v}=\mathbf{S}_{s} \mathbf{c}_{v}
\end{aligned}
$$

where the sample matrix $\mathbf{S}_{s}$ is defined as

$$
\left\{\mathbf{S}_{s}\right\}_{i, N_{g} l+k}=\beta^{n}\left(\frac{x_{i}}{a}-k\right) \beta^{n}\left(\frac{y_{i}}{a}-l\right) .
$$

Let $\mathbf{W}_{x}$ and $\mathbf{W}_{y}$ be the diagonal matrices defined by

$$
\begin{aligned}
& \left\{\mathbf{W}_{x}\right\}_{i i}=d_{x i} \\
& \left\{\mathbf{W}_{y}\right\}_{i i}=d_{y i}
\end{aligned}
$$

where $d_{x i}$ and $d_{y i}$ are the components of $\mathbf{d}_{i}$. Further, let

$$
\mathbf{t}_{v d}=\left[\cdots \mathbf{d}_{i}^{T} \mathbf{v}\left(\mathbf{x}_{i}\right) \cdots\right]^{T}
$$

It can be verified that

$$
\mathbf{t}_{v d}=\mathbf{W}_{x} \mathbf{t}_{u}+\mathbf{W}_{y} \mathbf{t}_{v}=\mathbf{W}_{x} \mathbf{S} \mathbf{c}_{u}+\mathbf{W}_{y} \mathbf{S c}_{v} .
$$

We now define

$$
\begin{aligned}
\mathbf{W} & =\left[\begin{array}{ll}
\mathbf{W}_{x} & \mathbf{W}_{y}
\end{array}\right] \\
\mathbf{S} & =\left[\begin{array}{cc}
\mathbf{S}_{s} & \mathbf{0} \\
\mathbf{0} & \mathbf{S}_{s}
\end{array}\right] .
\end{aligned}
$$

Then, $\mathbf{t}_{v d}$ becomes

$$
\mathbf{t}_{v d}=\mathbf{W S c}
$$

Let $\mathbf{m}=\left[\cdots m_{i} \cdots\right]^{T}$. Putting all these elements together, we express $(5)$ in the following standard matrix form:

$$
\begin{aligned}
J_{\mathrm{LS}}(\mathbf{v}) & =\left\|\mathbf{t}_{v d}-\mathbf{m}\right\|^{2}=\|\mathbf{W S c}-\mathbf{m}\|^{2} \\
& =\mathbf{c}^{T} \mathbf{A}_{\mathrm{LS}} \mathbf{c}-2 \mathbf{c}^{T} \mathbf{B}^{T} \mathbf{m}+\mathbf{m}^{T} \mathbf{m}
\end{aligned}
$$

where

$$
\begin{aligned}
\mathbf{A}_{\mathrm{LS}} & =\mathbf{S}^{T} \mathbf{W}^{T} \mathbf{W S} \\
\mathbf{B} & =\mathbf{W S} .
\end{aligned}
$$

Note that $\mathbf{A}_{\mathrm{LS}}$ is a square matrix of size $2 N_{g}^{2}$. Finally, the least squares reconstruction-i.e., the minimizer of (9) - is given by the solution of the following equation:

$$
\mathbf{c}_{\mathrm{LS}}=\mathbf{A}_{\mathrm{LS}}^{-1} \mathbf{b}
$$

where $\mathbf{b}=\mathbf{B}^{T} \mathbf{m}$. In the case where $\mathbf{A}_{\mathrm{LS}}$ is not of full rank, we consider the minimum norm solution which is given by $\mathbf{c}_{\mathrm{LS}}=$ $\mathbf{A}_{\mathrm{LS}}^{\dagger} \mathbf{b}$, where $\mathbf{A}_{\mathrm{LS}}^{\dagger}$ is the generalized (Moore-Penrose) inverse of $\mathbf{A}_{\mathrm{LS}}$.

\section{B. Regularized Least Squares Method (RLS)}

The reconstruction in the regularized least squares methods is the minimizer of an extended cost functional; it is obtained by adding a smoothness functional to the original least squares criterion, i.e.,

$$
J_{\mathrm{RLS}}(\mathbf{v})=J_{\mathrm{LS}}(\mathbf{v})+\lambda R(\mathbf{v}) .
$$

A smoothness functional $R(\mathbf{v})$ that is frequently used for the estimation of deformation fields is the weighted sum of membrane spline and thin-plate spline regularizers [17], [18] applied to each component. Specifically, we have

$$
R(\mathbf{v})=\lambda_{1}\left(\mathcal{D}_{1}(u)+\mathcal{D}_{1}(v)\right)+\lambda_{2}\left(\mathcal{D}_{2}(u)+\mathcal{D}_{2}(v)\right)
$$

where

$$
\begin{aligned}
& \mathcal{D}_{1}(u) \\
& =\iint\left(\frac{\partial u(x, y)}{\partial x}\right)^{2}+\left(\frac{\partial u(x, y)}{\partial y}\right)^{2} d x d y \\
& \mathcal{D}_{2}(u) \\
& =\iint\left(\frac{\partial^{2} u(x, y)}{\partial x^{2}}\right)^{2}+2\left(\frac{\partial^{2} u(x, y)}{\partial x \partial y}\right)^{2}+\left(\frac{\partial^{2} u(x, y)}{\partial y^{2}}\right)^{2} d x d y
\end{aligned}
$$

with $\lambda_{1} \geq 0$ and $\lambda_{2} \geq 0$. It is important to note that this fairly standard regularizer does not have any coupled terms, meaning that it does not enforce any special relationship between velocity components. 
The main task now is to express $\mathcal{D}_{1}($.$) and \mathcal{D}_{2}($.$) in terms of$ the expansion coefficients. In the appendix, we show that these can be written down relatively simply in terms of digital filtering operations and $l_{2}$-inner products as given below

$$
\begin{aligned}
& \mathcal{D}_{1}(u)=\left\langle g(k, l) * c_{k, l}^{u}, \quad c_{k, l}^{u}\right\rangle_{l_{2}\left(\mathbb{Z}^{2}\right)} \\
& \mathcal{D}_{2}(u)=\left\langle l(k, l) * c_{k, l}^{u}, \quad c_{k, l}^{u}\right\rangle_{l_{2}\left(\mathbb{Z}^{2}\right)} .
\end{aligned}
$$

The digital filters $g$ and $l$ are conveniently characterized by their $z$-transforms $G\left(z_{1}, z_{2}\right)$ and $L\left(z_{1}, z_{2}\right)$. For $B$-splines they take the following form:

$$
\begin{aligned}
G\left(z_{1}, z_{2}\right)= & \left(z_{1}-2+z_{1}^{-1}\right) B^{2 n-1}\left(z_{1}\right) B^{2 n+1}\left(z_{2}\right) \\
& +B^{2 n+1}\left(z_{1}\right)\left(z_{2}-2+z_{2}^{-1}\right) B^{2 n-1}\left(z_{2}\right) \\
L\left(z_{1}, z_{2}\right)= & \frac{1}{a^{2}}\left[\left(z_{1}-2+z_{1}^{-1}\right)^{2} B^{2 n-3}\left(z_{1}\right) B^{2 n+1}\left(z_{2}\right)\right. \\
& +2\left(z_{1}-2+z_{1}^{-1}\right) \\
& B^{2 n-1}\left(z_{1}\right)\left(z_{2}-2+z_{2}^{-1}\right) B^{2 n-1}\left(z_{2}\right) \\
& \left.+B^{2 n+1}\left(z_{1}\right)\left(z_{2}-2+z_{2}^{-1}\right)^{2} B^{2 n-3}\left(z_{2}\right)\right]
\end{aligned}
$$

where $B^{n}(z)=\sum_{k \in \mathbb{Z}} \beta^{n}(k) z^{-1}$ denotes the $z$-transform of the discrete $B$-spline of degree $n$. Equations (17) and (18) can also be written as

$$
\begin{aligned}
& \mathcal{D}_{1}(u)=\mathbf{c}_{u}^{T} \mathbf{R}_{g} \mathbf{c}_{u} \\
& \mathcal{D}_{2}(u)=\mathbf{c}_{u}^{T} \mathbf{R}_{l} \mathbf{c}_{u}
\end{aligned}
$$

where $\mathbf{R}_{g}$ and $\mathbf{R}_{l}$ are the circulant matrices corresponding to $G\left(z_{1}, z_{2}\right)$ and $L\left(z_{1}, z_{2}\right)$, respectively. This together with (9) yields the following expression for $J_{\mathrm{RLS}}(\mathbf{v})$ :

$$
\begin{aligned}
J_{\mathrm{RLS}}(\mathbf{v})=\mathbf{c}^{T} \mathbf{A}_{\mathrm{LS}} \mathbf{c}-2 \mathbf{c}^{T} \mathbf{B}^{T} \mathbf{m}+\mathbf{m}^{T} \mathbf{m} & \\
& +\lambda_{1} \mathbf{c}^{T} \mathbf{R}_{g e} \mathbf{c}+\lambda_{2} \mathbf{c}^{T} \mathbf{R}_{l e} \mathbf{c}
\end{aligned}
$$

where

$$
\begin{aligned}
\mathbf{R}_{g e} & =\left[\begin{array}{cc}
\mathbf{R}_{g} & \mathbf{0} \\
\mathbf{0} & \mathbf{R}_{g}
\end{array}\right] \\
\mathbf{R}_{l e} & =\left[\begin{array}{cc}
\mathbf{R}_{l} & \mathbf{0} \\
\mathbf{0} & \mathbf{R}_{l}
\end{array}\right] .
\end{aligned}
$$

Thus, the minimizer of (23) can be formally expressed as

$$
\mathbf{c}_{\mathrm{RLS}}=\mathbf{A}_{\mathrm{RLS}}^{-1} \mathbf{b},
$$

where $\mathbf{A}_{\mathrm{RLS}}=\mathbf{A}_{\mathrm{LS}}+\lambda_{1} \mathbf{R}_{g e}+\lambda_{2} \mathbf{R}_{l e}$. In practice, we do not compute the inverse but we solve the system using direct or iterative methods that are efficient for sparse systems.

\section{Vector Regularized Least Squares (VRLS)}

We now introduce the concept of a vector regularization that relies on an extended smoothness functional that couples the velocity components. The criterion to minimize is

$$
J_{\mathrm{VRLS}}(\mathbf{v})=J_{\mathrm{LS}}(\mathbf{v})+\lambda R_{E}(\mathbf{v})
$$

where $R_{E}(\mathbf{v})$ is our extended smoothness functional. The functional, $R_{E}(\mathbf{v})$, is based on the divergence and curl of the velocity field, which are defined as

$$
\begin{aligned}
\operatorname{Div} \mathbf{v}(\mathbf{x}) & =\partial_{x} u(x, y)+\partial_{y} v(x, y), \\
\operatorname{Curl} \mathbf{v}(\mathbf{x}) & =-\partial_{y} u(x, y)+\partial_{x} v(x, y) .
\end{aligned}
$$

The divergence of the velocity field quantifies the rate change of the density of the medium at a given point. The curl of a velocity field, on the other hand, is equal to twice the angular velocity within an infinitesimal neighborhood at the point of interest. We construct $R_{E}(\mathbf{v})$ as given below

$$
\begin{aligned}
R_{E}(\mathbf{v})= & \lambda_{d_{0}} \int(\operatorname{Div} \mathbf{v}(\mathbf{x}))^{2} d \mathbf{x} \\
& +\lambda_{d_{1}} \int\|\nabla(\operatorname{Div} \mathbf{v}(\mathbf{x}))\|^{2} d \mathbf{x} \\
& +\lambda_{c_{0}} \int(\operatorname{Curl} \mathbf{v}(\mathbf{x}))^{2} d \mathbf{x} \\
& +\lambda_{c_{1}} \int\|\nabla(\operatorname{Curl} \mathbf{v}(\mathbf{x}))\|^{2} d \mathbf{x} .
\end{aligned}
$$

Since the divergence gives the density change, the first term in the (27) quantifies the overall compression rate, whereas the second term gives the spatial roughness of this compression rate. Both terms are related to the deformation of the medium. The third term sums up the squared angular velocity. It does not directly quantify the deformation, but will tend to penalize rotations including rigid ones. The last term, on the other hand, is indeed a measure of deformation, as it captures the spatial variation of the angular velocity.

The idea behind using this regularizer is that it includes every derivative-based quantity that has a direct physical interpretation. This allows one to incorporate some a priori knowledge of the type of velocity field and also to specify physically plausible solutions. Interestingly, we have verified that any rotationally invariant functional of order lesser or equal to two takes the form (27). The proof will be published elsewhere.

As shown in the Appendix, the various terms in (27) can be written as

$$
\begin{aligned}
\int(\operatorname{Div} \mathbf{v}(\mathbf{x}))^{2} d \mathbf{x}= & \left\langle r_{11}(k, l) * c_{k, l}^{u}, \quad c_{k, l}^{u}\right\rangle \\
& +\left\langle r_{12}(k, l) * c_{k, l}^{v}, \quad c_{k, l}^{v}\right\rangle \\
& +2\left\langle r_{13}(k, l) * c_{k, l}^{u}, \quad c_{k, l}^{v}\right\rangle \\
\int(\operatorname{Curl} \mathbf{v}(\mathbf{x}))^{2} d \mathbf{x}= & \left\langle r_{11}(k, l) * c_{k, l}^{u}, \quad c_{k, l}^{u}\right\rangle \\
& +\left\langle r_{12}(k, l) * c_{k, l}^{v}, \quad c_{k, l}^{v}\right\rangle \\
& -2\left\langle r_{13}(k, l) * c_{k, l}^{u}, \quad c_{k, l}^{v}\right\rangle \\
\int\|\nabla(\operatorname{Div} \mathbf{v}(\mathbf{x}))\|^{2} d \mathbf{x}= & \left\langle r_{21}(k, l) * c_{k, l}^{u}, \quad c_{k, l}^{u}\right\rangle \\
& +\left\langle r_{22}(k, l) * c_{k, l}^{v}, \quad c_{k, l}^{v}\right\rangle \\
& +2\left\langle r_{23}(k, l) * c_{k, l}^{u}, \quad c_{k, l}^{v}\right\rangle \\
\int\|\nabla(\operatorname{Curl} \mathbf{v}(\mathbf{x}))\|^{2} d \mathbf{x}= & \left\langle r_{21}(k, l) * c_{k, l}^{u}, \quad c_{k, l}^{u}\right\rangle \\
& +\left\langle r_{22}(k, l) * c_{k, l}^{v}, \quad c_{k, l}^{v}\right\rangle \\
& -2\left\langle r_{23}(k, l) * c_{k, l}^{u}, \quad c_{k, l}^{v}\right\rangle
\end{aligned}
$$


where the underlying digital filters are defined as follows:

$$
\begin{aligned}
& R_{11}\left(z_{1}, z_{2}\right)=\left(z_{1}-2+z_{1}^{-1}\right) \\
& B^{2 n-1}\left(z_{1}\right) B^{2 n+1}\left(z_{2}\right) \\
& R_{12}\left(z_{1}, z_{2}\right)= B^{2 n+1}\left(z_{1}\right) \\
&\left(z_{2}-2+z_{2}^{-1}\right) B^{2 n-1}\left(z_{2}\right) \\
& R_{13}\left(z_{1}, z_{2}\right)= D^{2 n}\left(z_{1}\right) D^{2 n}\left(z_{2}^{-1}\right) \\
& R_{21}\left(z_{1}, z_{2}\right)= \frac{1}{a^{2}}\left[\left(z_{1}-2+z_{1}^{-1}\right)^{2}\right. \\
& B^{2 n-3}\left(z_{1}\right) B^{2 n+1}\left(z_{2}\right) \\
&+\left(z_{1}-2+z_{1}^{-1}\right) B^{2 n-1}\left(z_{1}\right) \\
&\left.\left(z_{2}-2+z_{2}^{-1}\right) B^{2 n-1}\left(z_{2}\right)\right] \\
& R_{22}\left(z_{1}, z_{2}\right)= \frac{1}{a^{2}}\left[B^{2 n+1}\left(z_{1}\right)\right. \\
&\left(z_{2}-2+z_{2}^{-1}\right)^{2} B^{2 n-3}\left(z_{2}\right) \\
&+\left(z_{1}-2+z_{1}^{-1}\right) B^{2 n-1}\left(z_{1}\right) \\
&\left.\left(z_{2}-2+z_{2}^{-1}\right) B^{2 n-1}\left(z_{2}\right)\right] \\
& \frac{1}{a^{2}}\left[\left(z_{1}-2+z_{1}^{-1}\right)\right. \\
& D^{2 n-2}\left(z_{1}\right) D^{2 n}\left(z_{2}^{-1}\right) \\
&+D^{2 n}\left(z_{1}\right) \\
&\left.\left(z_{2}-2+z_{2}^{-1}\right) D^{2 n-2}\left(z_{2}^{-1}\right)\right] . \\
& R_{23}\left(z_{1}, z_{2}\right)
\end{aligned}
$$

Here, $D^{n}(z)=\sum_{k \in \mathbb{Z}}\left(\beta^{n}(x+1 / 2)-\beta^{n}(x-1 / 2)\right) z^{-1}$. In matrix form, (28)-(31) read

$$
\begin{aligned}
\int(\operatorname{Div} \mathbf{v}(\mathbf{x}))^{2} d \mathbf{x}= & \mathbf{c}_{u}^{T} \mathbf{R}_{11} \mathbf{c}_{u}+\mathbf{c}_{v}^{T} \mathbf{R}_{12} \mathbf{c}_{v} \\
& +2 \mathbf{c}_{u}^{T} \mathbf{R}_{13} \mathbf{c}_{v} \\
\int(\operatorname{Curl} \mathbf{v}(\mathbf{x}))^{2} d \mathbf{x}= & \mathbf{c}_{u}^{T} \mathbf{R}_{12} \mathbf{c}_{u}+\mathbf{c}_{v}^{T} \mathbf{R}_{11} \mathbf{c}_{v} \\
& -2 \mathbf{c}_{u}^{T} \mathbf{R}_{13} \mathbf{c}_{v} \\
\int\|\nabla(\operatorname{Div} \mathbf{v}(\mathbf{x}))\|^{2} d \mathbf{x}= & \mathbf{c}_{u}^{T} \mathbf{R}_{21} \mathbf{c}_{u}+\mathbf{c}_{v}^{T} \mathbf{R}_{22} \mathbf{c}_{v} \\
& +2 \mathbf{c}_{u}^{T} \mathbf{R}_{23} \mathbf{c}_{v} \\
\int\|\nabla(\operatorname{Curl} \mathbf{v}(\mathbf{x}))\|^{2} d \mathbf{x}= & \mathbf{c}_{u}^{T} \mathbf{R}_{22} \mathbf{c}_{u}+\mathbf{c}_{v}^{T} \mathbf{R}_{21} \mathbf{c}_{v} \\
& -2 \mathbf{c}_{u}^{T} \mathbf{R}_{23} \mathbf{c}_{v} .
\end{aligned}
$$

The cost functional, $J_{\mathrm{VRLS}}(\mathbf{v})$, now becomes

$$
\begin{aligned}
J_{\mathrm{VRLS}}(\mathbf{v})= & \mathbf{c}^{T} \mathbf{A}_{\mathrm{LS}} \mathbf{c}-2 \mathbf{c}^{T} \mathbf{B}^{T} \mathbf{m}+\mathbf{m}^{T} \mathbf{m} \\
& +\lambda_{d_{0}} \mathbf{c}^{T} \mathbf{R}_{d_{0}} \mathbf{c}+\lambda_{c_{0}} \mathbf{c}^{T} \mathbf{R}_{c_{0}} \mathbf{c} \\
& +\lambda_{d_{1}} \mathbf{c}^{T} \mathbf{R}_{d_{1}} \mathbf{c}+\lambda_{c_{1}} \mathbf{c}^{T} \mathbf{R}_{d_{1}} \mathbf{c}
\end{aligned}
$$

where

$$
\begin{aligned}
& \mathbf{R}_{d_{0}}= {\left[\begin{array}{ll}
\mathbf{R}_{11} & \mathbf{R}_{13} \\
\mathbf{R}_{13} & \mathbf{R}_{12}
\end{array}\right] } \\
& \mathbf{R}_{c_{0}}= {\left[\begin{array}{cc}
\mathbf{R}_{12} & -\mathbf{R}_{13} \\
-\mathbf{R}_{13} & \mathbf{R}_{11}
\end{array}\right] } \\
& \mathbf{R}_{d_{1}}=\left[\begin{array}{ll}
\mathbf{R}_{21} & \mathbf{R}_{23} \\
\mathbf{R}_{23} & \mathbf{R}_{22}
\end{array}\right] \\
& \mathbf{R}_{c_{1}}=\left[\begin{array}{cc}
\mathbf{R}_{22} & -\mathbf{R}_{23} \\
-\mathbf{R}_{23} & \mathbf{R}_{21}
\end{array}\right] .
\end{aligned}
$$

Note that the functional, $R_{E}(\mathbf{v})$, includes $R(\mathbf{v})$ as a special case. To show this, we first observe from (32), (33), and (19) that

$$
G\left(z_{1}, z_{2}\right)=R_{11}\left(z_{1}, z_{2}\right)+R_{12}\left(z_{1}, z_{2}\right)
$$

Similarly, using (35), (36), and (20), we get

$$
L\left(z_{1}, z_{2}\right)=R_{21}\left(z_{1}, z_{2}\right)+R_{22}\left(z_{1}, z_{2}\right) .
$$

These two relations in turn imply

$$
\begin{aligned}
& \mathbf{R}_{d_{0}}+\mathbf{R}_{c_{0}}=\mathbf{R}_{g e} \\
& \mathbf{R}_{d_{1}}+\mathbf{R}_{c_{1}}=\mathbf{R}_{l e} .
\end{aligned}
$$

Thus, we conclude that $R_{E}(\mathbf{v})=R(\mathbf{v})$ if $\lambda_{d_{0}}=\lambda_{c_{0}}=\lambda_{1}$ and $\lambda_{d_{1}}=\lambda_{c_{1}}=\lambda_{2}$. In fact, it can be shown that this relation is valid for any vector function and not just for those that are included in our $B$-spline reconstruction space.

Finally, the solution for the VRLS method-i.e., the minimizer of (38) — is given by

$$
\mathbf{c}_{\mathrm{VRLS}}=\mathbf{A}_{\mathrm{VRLS}}^{-1} \mathbf{b}
$$

where

$$
\mathbf{A}_{\mathrm{VRLS}}=\mathbf{A}_{\mathrm{LS}}+\lambda_{d_{0}} \mathbf{R}_{d_{0}}+\lambda_{c_{0}} \mathbf{R}_{c_{0}}+\lambda_{d_{1}} \mathbf{R}_{d_{1}}+\lambda_{c_{1}} \mathbf{R}_{c_{1}} .
$$

Note that this kind of regularized reconstruction method that involves the projected sampling model has been proposed for velocity field reconstruction from tagged MRI data [19]. However, the approach of Suter et al. differs from the proposed method in two respects: 1) it does not involve any physics based regularization; 2) the method involves the computation of globally supported functions which is computationally expensive, whereas the proposed method is tailored for computational efficiency, as will be demonstrated in the subsequent sections.

\section{Structure of the Linear System of Equations}

Let us now have a closer look at the structure of the matrices involved and make a few comments on the numerical methods for solving the linear system of equations. We only consider the linear system that correspond to VRLS method since the other methods are special cases of the VRLS method. It can be shown that the matrix given in (43) can be express as

$$
\begin{aligned}
\mathbf{A}_{\mathrm{VRLS}} & =\left[\begin{array}{cc}
\mathbf{A}_{u} & \mathbf{A}_{u v} \\
\mathbf{A}_{u v} & \mathbf{A}_{v}
\end{array}\right] \\
& =\left[\begin{array}{cc}
\mathbf{D}_{u} & \mathbf{D}_{u v} \\
\mathbf{D}_{u v} & \mathbf{D}_{v}
\end{array}\right]+\left[\begin{array}{cc}
\mathbf{R}_{u} & \mathbf{R}_{u v} \\
\mathbf{R}_{u v} & \mathbf{R}_{v}
\end{array}\right]
\end{aligned}
$$

where

$$
\begin{aligned}
& \left\{\mathbf{D}_{u}\right\}_{N_{g} k+j, N_{g} m+l} \\
& \quad=\sum_{i} d_{x i}^{2} \beta^{n}\left(\frac{x_{i}}{a}-j\right) \beta^{n}\left(\frac{y_{i}}{a}-k\right) \beta^{n}\left(\frac{x_{i}}{a}-l\right) \beta^{n}\left(\frac{y_{i}}{a}-m\right)
\end{aligned}
$$




$$
\begin{aligned}
& \left\{\mathbf{D}_{v}\right\}_{N_{g} k+j, N_{g} m+l} \\
& \quad=\sum_{i} d_{y i}^{2} \beta^{n}\left(\frac{x_{i}}{a}-j\right) \beta^{n}\left(\frac{y_{i}}{a}-k\right) \beta^{n}\left(\frac{x_{i}}{a}-l\right) \beta^{n}\left(\frac{y_{i}}{a}-m\right) \\
& \left\{\mathbf{D}_{u v}\right\}_{N_{g} k+j, N_{g} m+l} \\
& \quad=\sum_{i} d_{x i} d_{y i} \beta^{n}\left(\frac{x_{i}}{a}-j\right) \beta^{n}\left(\frac{y_{i}}{a}-k\right) \beta^{n}\left(\frac{x_{i}}{a}-l\right) \beta^{n}\left(\frac{y_{i}}{a}-m\right)
\end{aligned}
$$

and $\mathbf{R}_{u}, \mathbf{R}_{v}$, and $\mathbf{R}_{u v}$ are the circulant matrices corresponding to some filters $R_{u}\left(z_{1}, z_{2}\right), R_{v}\left(z_{1}, z_{2}\right)$, and $R_{u v}\left(z_{1}, z_{2}\right)$, respectively. The filters are given by

$$
\begin{aligned}
R_{u}\left(z_{1}, z_{2}\right)= & \lambda_{d_{0}} R_{11}\left(z_{1}, z_{2}\right)+\lambda_{c_{0}} R_{12}\left(z_{1}, z_{2}\right) \\
& +\lambda_{d_{1}} R_{21}\left(z_{1}, z_{2}\right)+\lambda_{c_{1}} R_{22}\left(z_{1}, z_{2}\right) \\
R_{v}\left(z_{1}, z_{2}\right)= & \lambda_{d_{0}} R_{12}\left(z_{1}, z_{2}\right)+\lambda_{c_{0}} R_{11}\left(z_{1}, z_{2}\right) \\
& +\lambda_{d_{1}} R_{22}\left(z_{1}, z_{2}\right)+\lambda_{c_{1}} R_{21}\left(z_{1}, z_{2}\right) \\
R_{u v}\left(z_{1}, z_{2}\right)= & \left(\lambda_{d_{0}}-\lambda_{c_{0}}\right) R_{13}\left(z_{1}, z_{2}\right) \\
& +\left(\lambda_{d_{1}}-\lambda_{c_{1}}\right) R_{23}\left(z_{1}, z_{2}\right) .
\end{aligned}
$$

The above matrices are of size $N_{g}^{2} \times N_{g}^{2}$. From (45)-(47), it can be observed that the sample matrices $\mathbf{D}_{u}, \mathbf{D}_{v}$, and $\mathbf{D}_{u v}$ are sparse. In particular, assuming that each grid interval has a sample point (worst case sparsity), it can be verified that an element $\{\cdot\}_{N_{g} k+j, N_{g} m+l}$ is zero for $\max (|l-j|,|m-k|)>n$ (finite support of $B$-splines). Hence, the matrices are block-band diagonal of width $2 n+1$ and each of the constituent block of size $N_{g} \times N_{g}$ is also band diagonal of width $2 n+1$. Moreover, these matrices are symmetric and each constituent block is symmetric as well. Next, from the expression (32)-(37), the filters are symmetric and of length $2 n+1$. This implies that the matrices $\mathbf{R}_{u}, \mathbf{R}_{v}$, and $\mathbf{R}_{u v}$ have the same structure as that of the sample matrices (with the additional property of being circulant). As a result, the matrices $\mathbf{A}_{u}, \mathbf{A}_{v}$, and $\mathbf{A}_{u v}$ also have the same sparsity and symmetry.

Hence, the overall matrix $\mathbf{A}_{\mathrm{VRLS}}$ is sparse and symmetric. Since $\mathbf{A}_{\mathrm{VRLS}}$ is typically nonsingular as in any regularized linear problem, it is positive definite.

It can be shown that the RHS of the linear system of equations is given by

$$
\mathbf{b}=\left[\begin{array}{l}
\mathbf{b}_{u} \\
\mathbf{b}_{v}
\end{array}\right]
$$

where

$$
\begin{aligned}
\left\{\mathbf{b}_{u}\right\}_{N_{g} k+j} & =\sum_{i} d_{x i} \beta^{n}\left(\frac{x_{i}}{a}-j\right) \beta^{n}\left(\frac{y_{i}}{a}-k\right) m_{i} \\
\left\{\mathbf{b}_{v}\right\}_{N_{g} k+j} & =\sum_{i} d_{y i} \beta^{n}\left(\frac{x_{i}}{a}-j\right) \beta^{n}\left(\frac{y_{i}}{a}-k\right) m_{i} .
\end{aligned}
$$

We now consider an important point on the ordering of coefficients $\left\{c_{k, l}^{u}\right\}$ and $\left\{c_{k, l}^{v}\right\}$ to get the coefficient vector $\mathbf{c}$. Until now, we used the concatenated ordering $\mathbf{c}=\left[\cdots c_{k, l}^{u} \ldots c_{k, l}^{v} \ldots\right]^{T}$ to facilitate the derivations. However, there is an alternative ordering that is physically more meaningful

$$
\mathbf{c}=\left[\cdots c_{k, l}^{u} c_{k, l}^{v} \ldots\right]^{T} .
$$

The corresponding linear system of equations is given by

$$
\hat{\mathbf{A}} \mathbf{c}=\hat{\mathbf{b}}
$$

where

$$
\begin{aligned}
\hat{\mathbf{A}}= & \left(\mathbf{A}_{u} \oplus\left[\begin{array}{ll}
1 & 0 \\
0 & 0
\end{array}\right]\right)+\left(\mathbf{A}_{v} \oplus\left[\begin{array}{ll}
0 & 0 \\
0 & 1
\end{array}\right]\right) \\
& +\left(\mathbf{A}_{u v} \oplus\left[\begin{array}{ll}
0 & 1 \\
1 & 0
\end{array}\right]\right) \\
\hat{\mathbf{b}}= & \mathbf{b}_{u} \oplus\left[\begin{array}{l}
1 \\
0
\end{array}\right]+\mathbf{b}_{v} \oplus\left[\begin{array}{l}
0 \\
1
\end{array}\right] .
\end{aligned}
$$

The main advantage of this modified linear system is that the matrix $\hat{\mathbf{A}}$ retains the sparse structure of its constituent matrices $\mathbf{A}_{u}, \mathbf{A}_{v}$, and $\mathbf{A}_{u v}$ with $2 \times 2$ matrices playing the role of the scalars elements in the latter ones. In this configuration, the nonzero elements are all concentrated near the diagonal. This allows us to conclude that the solution can be obtained by solving a sparse, well-conditioned, diagonally-dominant, and positivedefinite linear system of equations. Hence, we have at our disposal a rich set of computationally efficient iterative methods to achieve the required reconstruction. This is the primary advantage of the proposed scheme over an analytical method such as the one presented in [19].

\section{E. Computational Complexity}

The computational task essentially consists of two parts: 1) constructing the linear system of equations, 2) and solving the system. The first is much less demanding than the second.

The first part is essentially the computation of the matrices $\mathbf{D}_{u}, \mathbf{D}_{v}$, and $\mathbf{D}_{u v}$ using (45)-(47) and then adding the circulent matrices. The latter is negligible whereas the former is proportional to $N(n+1)$ where $N$ is the number of samples points.

The second part, which is the computation of the solution, has a complexity that is proportional to the number of nonredundant and nonzero elements in $\hat{\mathbf{A}}_{\text {VRLS. }}$ Because of the symmetry and the sparsity properties identified before, the number of nonzero elements is equal to $\left(N_{g}(n+1)\right)^{2}$. Hence, the complexity of solving the linear system is proportional to $[(n+1) / a]^{2}$. On the other hand, since the approximation error $B$ for the spline discretization is $\mathcal{O}\left(a^{n+1}\right)$, the reconstruction accuracy is proportional to $a^{n+1}$. As a consequence, $a$ becomes a trade-off factor that allows a compromise between the computational complexity and the reconstruction accuracy. In this regard, $B$-splines give the best trade-off since they have the highest approximation order for a given support size [20] (support size determines the computational complexity). 


\section{EXPERIMENTS}

To demonstrate the feasibility of the proposed formulation and to compare different methods, we first study the reconstruction of synthetic data. We then move to real phantom data, where the motion is controlled and known a priori. Finally, we present reconstruction results obtained from two types of clinical data for the assessment of: 1) blood flow in the carotid bifurcation, and 2) cardiac wall motion. We use a Gaussian noise model in our experiments, since the noise in Doppler systems is usually found to be Gaussian [1].

\section{A. Tuning the Parameters of the Algorithm}

We tune the regularization parameters by a two-step procedure. First, the parameter search space is constrained by incorporating some a priori knowledge on the type motion if available. Next, by performing multiple reconstructions, the parameters are updated by an iterative search within the constrained search space such that the difference between the data term of the cost functional $J_{\mathrm{LS}}$ and the noise variance is minimum. In other words, the parameters are obtained as the minimizer of the following cost:

$$
\mathcal{C}=\frac{\left|J_{\mathrm{LS}}\left(\mathbf{v}_{r}\right)-\sigma^{2}\right|}{\sigma^{2}}
$$

where $\mathbf{v}_{r}(\mathbf{x})$ is the reconstructed velocity field, and $\sigma^{2}$ is the noise variance. The idea behind this strategy is that, if the reconstruction function coincides with the underlying true velocity field, then the error in the data term is entirely due to noise implying that $J_{\mathrm{LS}}\left(\mathbf{v}_{r}\right) \rightarrow \sigma^{2}$.

Since $J_{\mathrm{LS}}\left(\mathbf{v}_{r}\right)$ is an increasing function with respect to each regularization parameter, we can achieve a faster convergence if we keep the parameters proportional to $\sigma^{2}$.

\section{B. Validating the Reconstructed Function}

While it is easy to validate the reconstructed field in a test case where the ground truth is known, it is less straightforward to do so in a clinical scenario. Here, we propose a cross-validation strategy for the quantitative assessment of our method. The idea is to use only part of the available data for field reconstruction by the above mentioned method (including the iterative search for optimal parameter determination). We then evaluate (51) using the data that was left out from the reconstruction process. If the cost (51) is sufficiently close to the minimum attained during the iterative reconstruction, then we are able to conclude that the reconstructed field is consistent with the full data set, suggesting that it is a good approximation of the underlying true velocity field.

\section{Synthetic Rotating Phantom}

Here we consider the vector field scenario described in Section II-B, which corresponds to a planar rotation with a constant angular velocity. We demonstrated earlier that the single probe measurement set is ambiguous and does not permit the recovery of the motion parameters. Hence we consider measurements from two probe locations, since it is the only practical way to resolve the ambiguity. We study the performance of all three method on such a measurement set.

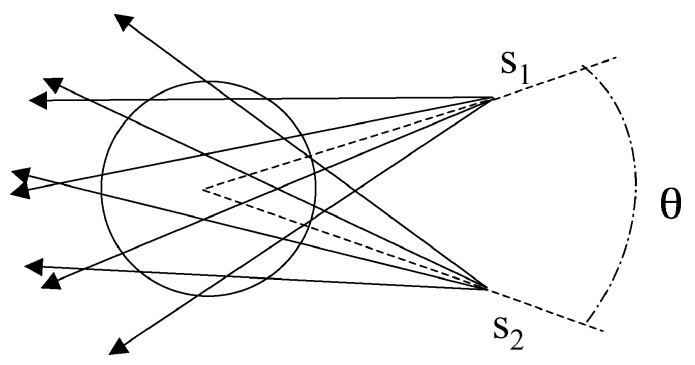

Fig. 2. Schematic for synthetic phantom experiment.

The required Doppler data is simulated for two sector scan probes. Let $\mathbf{s}_{1}=\left[\begin{array}{ll}x_{1} & y_{1}\end{array}\right]^{T}$ and $\mathbf{s}_{2}=\left[\begin{array}{ll}x_{2} & y_{2}\end{array}\right]^{T}$ be the locations, and let $\theta$ be the angle subtended by the probe locations with respect to the center of rotation (Fig. 2). The idealized phantom data is now obtained by sampling two functions $v_{1}(\mathbf{x})$ and $v_{2}(\mathbf{x})$, where

$$
\begin{aligned}
v_{j}(\mathbf{x}) & =v_{j}(x, y) \\
& =\mathbf{d}_{j}^{T}(\mathbf{x}) \mathbf{v}(\mathbf{x}) \\
& =\left[d_{x_{j}}(x, y) d_{y_{j}}(x, y)\right]\left[\begin{array}{l}
\left.u_{(} x, y\right) \\
\left.v_{(} x, y\right)
\end{array}\right] .
\end{aligned}
$$

Here, $u(x, y)=-\omega\left(y-y_{c}\right), v(x, y)=\omega\left(x-x_{c}\right)$, and

$$
\begin{aligned}
& d_{x_{j}}(x, y)=\frac{x-x_{j}}{\sqrt{\left(x-x_{j}\right)^{2}+\left(y-y_{j}\right)^{2}}} \\
& d_{y_{j}}(x, y)=\frac{y-y_{j}}{\sqrt{\left(x-x_{j}\right)^{2}+\left(y-y_{j}\right)^{2}}} .
\end{aligned}
$$

We are also adding noise to make the problem more realistic. Each of the functions $v_{1}(\mathbf{x})$ and $v_{2}(\mathbf{x})$ are uniformly sampled with respect to the polar coordinate systems with origins $\mathbf{s}_{1}$, and $\mathbf{S}_{2}$, respectively. However, note that the samples are nonuniform with respect to our cartesian reconstruction system. Let $\left\{\mathbf{x}_{j i}, i=1, \ldots, N\right\}$ be the set of sample locations for the probe $\mathbf{s}_{j}$. The input for the algorithm is given by the following list of triplets:

$$
\left\{\mathbf{x}_{j i}, \mathbf{d}_{j i}, m_{j i}, j=1,2 ; i=1, \ldots, N\right\}
$$

where $\mathbf{d}_{j i}=\mathbf{d}_{j}\left(\mathbf{x}_{j i}\right)$, and $m_{j i}=\mathbf{d}_{j}^{T}\left(\mathbf{x}_{j i}\right) \mathbf{v}\left(\mathbf{x}_{j i}\right)+n_{j i}$ with $n_{j i}$ being some independent and identically distributed (i.i.d.) Gaussian measurement noise with variance $\sigma^{2}$. Note that the richness of the data set is controlled by the magnitude of $\theta$, and that the reconstruction becomes easier as $\theta$ tends to $90^{\circ}$.

Now we consider the choice of regularization parameters. For a rigid rotation, the divergence is zero. The gradient of curl is zero as well, except at the boundaries. Hence, the appropriate setting will be $\left\{\lambda_{d_{0}} \rightarrow \infty, \lambda_{d_{1}}=0, \lambda_{c_{0}}=0, \lambda_{c_{1}} \rightarrow \infty\right\}$. However, since the gradient of curl is nonzero at the boundaries, the choice $\lambda_{c_{1}} \rightarrow \infty$ will lead to a sizable error therein. A good compromise can be achieved by choosing $\left\{\lambda_{d_{0}} \rightarrow \infty, \lambda_{d_{1}}=\right.$ $\left.0, \lambda_{c_{0}}=0, \lambda_{c_{1}}=k \sigma^{2}\right\}$, where $k$ is an adequately chosen positive real number. The constant $k$ is chosen iteratively to match the variance as described before. For the RLS method, we need to set $\lambda_{1}=0$, since a rigid rotation contains the 


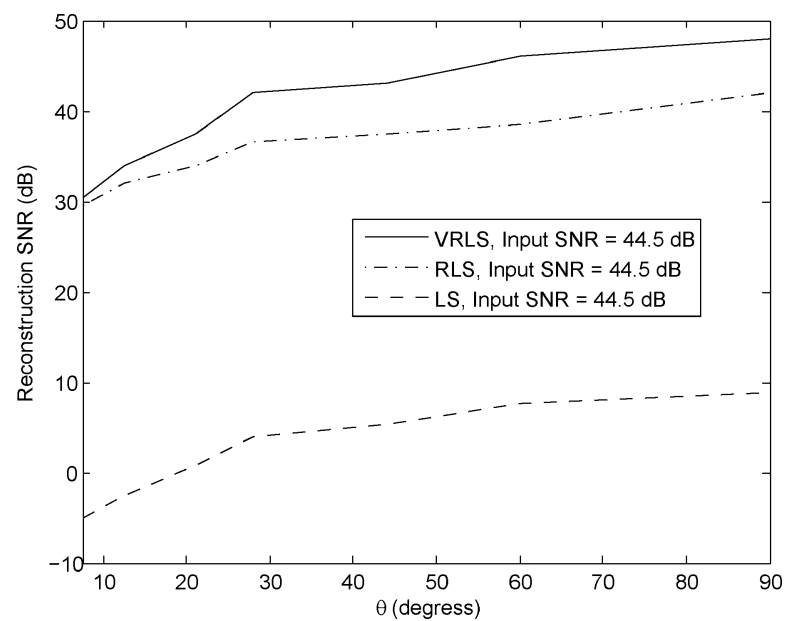

Fig. 3. Reconstruction error for synthetic phantom experiment.

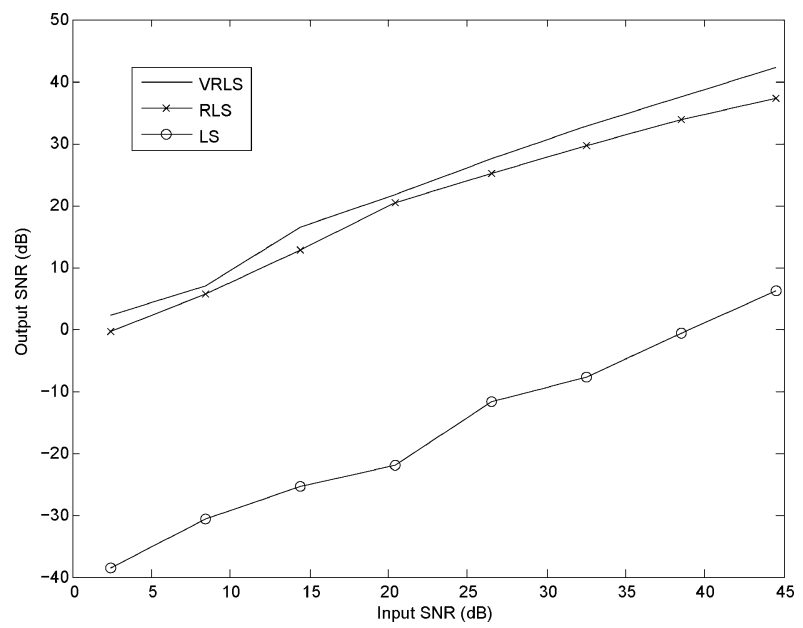

Fig. 4. Reconstruction error for synthetic phantom experiment.

first order spatial variation. Hence, the appropriate setting is $\left\{\lambda_{1}=0, \lambda_{2}=k \sigma^{2}\right\}$.

Before presenting our results, we define the following quantities:

$$
\begin{aligned}
\text { Input SNR } & =10 \times \log \left(\frac{E\left[\mathbf{d}_{j i}^{T} \mathbf{v}\left(\mathbf{x}_{j i}\right)^{2}\right]}{\sigma^{2}}\right) \\
\text { Recons. SNR } & =10 \times \log \left(E\left[\frac{\|\mathbf{v}(\mathbf{k})\|^{2}}{\left\|\mathbf{v}_{r}(\mathbf{k})-\mathbf{v}(\mathbf{k})\right\|^{2}}\right]\right)
\end{aligned}
$$

where $\mathbf{v}(\mathbf{x})$ is the true velocity field. Note that the input signal-to-noise ratio (SNR) is computed over the available sample locations, whereas the reconstruction SNR is computed over the reconstruction grid.

Fig. 3 gives reconstruction SNR for VRLS, RLS, and LS as a function of $\theta$, for input SNR equal to $44.5 \mathrm{~dB}$. It can be observed that the reconstruction error decreases with increasing $\theta$, as one expects. Also, one can clearly see the superiority of VRLS. Fig. 4 gives the reconstruction SNR for all three methods as a function of the input SNR with $\theta=30^{\circ}$. Note that VRLS performs the best, and that the reconstruction error for the conventional LS approach is far worse (off by more than $30 \mathrm{~dB}$ ). All

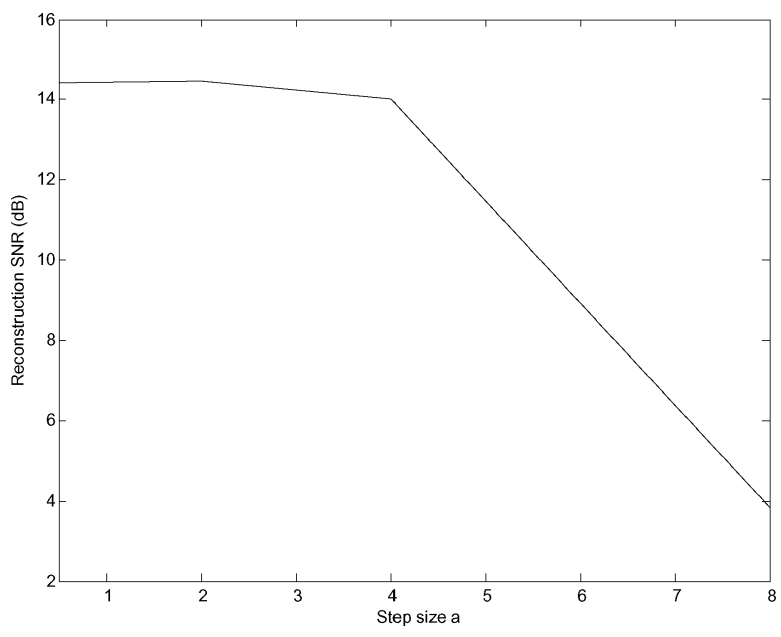

Fig. 5. VRLS Reconstruction error for synthetic phantom with nonrigid motion. Input $\mathrm{SNR}=10 \mathrm{~dB}$.

the reconstructions were done on a $16 \times 16$ grid with $a=4$ and with the number of samples equal to the number of grid points.

Note that when there is no input noise, the reconstruction error is dominated by the minimum amount of smoothing that is required to make the linear system of equations well conditioned.

\section{Synthetic Phantom With Nonrigid Motion}

In this experiment, we consider a more complex model for the synthetic phantom. Specifically, we adopt a nonrigid motion model given by

$$
\mathbf{v}(\mathbf{x})=\alpha\left[\begin{array}{l}
\frac{\partial}{\partial x} \varphi(\|\mathbf{x}\|) \\
\frac{\partial}{\partial y} \varphi(\|\mathbf{x}\|)
\end{array}\right]+\beta\left[\begin{array}{l}
\frac{-\partial}{\partial y} \varphi(\|\mathbf{x}\|) \\
\frac{\partial}{\partial x} \varphi(\|\mathbf{x}\|)
\end{array}\right]
$$

where $\varphi(r)=(1 / 2 \pi) e^{-r^{2} / 2}, \alpha \geq 0$, and $\beta \geq 0$. The first term is the curl-free component and the second is the divergencefree component. In continuum mechanics, they are identified as the irrotational and solenoidal components of the velocity field. In this experiment too, the input data are the measurements simulated for two sector scan probes (Fig. 2) with some i.i.d Gaussian noise.

Since the velocity field has a nonzero divergence and a nonzero curl, we have to choose $\lambda_{d_{0}}=\lambda_{c_{0}}=0$. The remaining parameters $\lambda_{d_{1}}$ and $\lambda_{c_{1}}$ are refined iteratively. Interestingly, we found that the resulting parameters satisfy

$$
\begin{aligned}
& \lambda_{d_{1}}=k \sigma^{2} \beta^{2} \\
& \lambda_{c_{1}}=k \sigma^{2} \alpha^{2}
\end{aligned}
$$

where $k$ is a suitable constant. In other words, the ratio $\lambda_{d_{1}} / \lambda_{c_{1}}$ is equal to the ratio of the energies of divergence-free and curlfree parts of the original velocity field.

The chosen range for the reconstruction grid is $[-3,3]$ (essential support of the Gaussian) and the average sampling density is 28 samples per unit area. Fig. 5 compares the reconstruction result for VRLS obtained for different values of the step size $a$ with $\theta=30^{\circ}, \alpha=0.25$, and $\beta=0.75$. The input SNR is $10 \mathrm{~dB}$. The reconstruction SNR is nearly constant when $a$ is sufficiently 


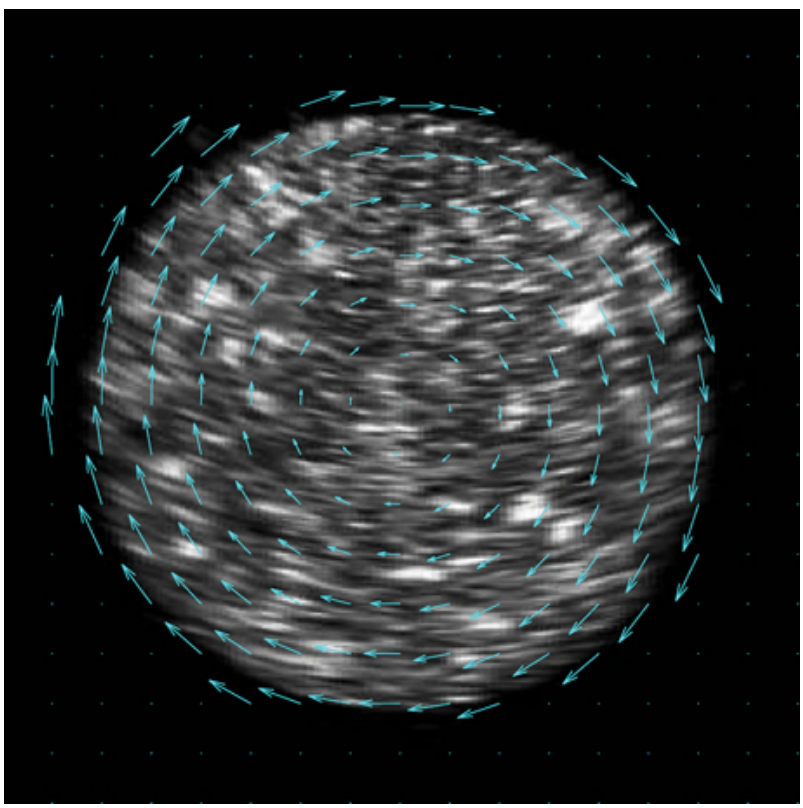

Fig. 6. Reconstructed motion field for the rotating real phantom.

small and falls off drastically when it becomes too large. For practical purpose, the choice $a=2$ offers a good compromise in terms of quality and computational cost $(\mathrm{SNR}=14.4 \mathrm{~dB})$.

We also performed reconstruction with two modified probe setting for $a=2: 1$ ) three views with the third view in the middle of the first two (angle between the outer views is $30^{\circ}$ and angle between adjacent views is $15^{\circ}$ ); 2) two views with angle $45^{\circ}$. The first modification yields an SNR of $15.2 \mathrm{~dB}$, whereas second yields $25.5 \mathrm{~dB}$. Qualitatively, we can say that the improvement in the first case is due to a better noise removal, whereas the improvement in the second is due to an increase in the amount of information, which is more significant than that of the first. We observed that, for the second, the SNR increases as the separation angle gets larger (the optimal angle being $90^{\circ}$. With some simplifying assumptions on the probe geometries, the reason for this behavior can be explained analytically for any type of velocity field. We are skipping this proof due to space limitations.

\section{E. Real Phantom Experiment}

The real phantom that we constructed for this experiment is a cylindrical tissue-mimicking object (gelatin) of radius 7.5 $\mathrm{cm}$ immersed in a water container. The object rotates with a constant angular velocity of $0.5 \mathrm{rad} / \mathrm{s}$. Doppler data were acquired using a sector scan probe, and reconstruction was performed from views differing by $10^{\circ}$. The regularization parameters were tuned in the same way as in the synthetic phantom experiment. Fig. 6 shows one frame of the $B$-mode intensity image with the superimposed reconstructed motion field. Since we know the true velocity field, we can compute the ideal measurements at the sample locations. Hence, we can compute the input noise variance, which was found to be $14.27 \mathrm{~dB}$. The reconstruction SNR for the VRLS method is $14.67 \mathrm{~dB}$. The average angular reconstruction error is $3^{\circ}$. The average radial velocity in the reconstructed velocity field is about $3.5 \%$ of the peak velocity in the true velocity field.

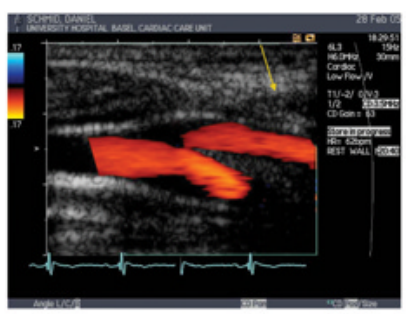

(a) $\alpha=70^{\circ}$

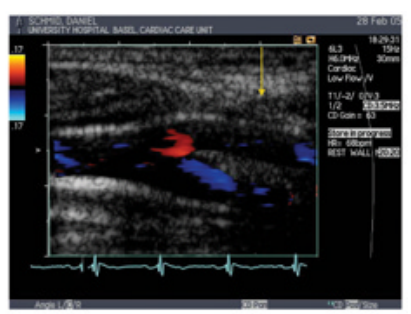

(b) $\alpha=90^{\circ}$

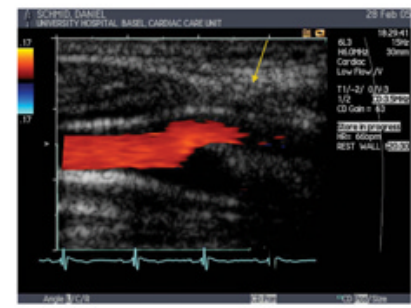

(c) $\alpha=110^{\circ}$

Fig. 7. Selected color Doppler images of carotid bifurcation. (a) $\alpha=70^{\circ}$. (b) $\alpha=90^{\circ}$. (c) $\alpha=110^{\circ}$.

\section{F. Blood Flow in Carotid Bifurcation}

Blood flow patterns in the carotid bifurcation have been reported to have significant influence on the development of artherosclerosis [21] which is a leading cause of heart attack and stroke. In this experiment, we attempted to reconstruct the complete blood-flow distribution in the carotid bifurcation from multiple-view Doppler data acquired using a parallel-beam probe setup with steering capability. We performed three acquisitions with beam angles $\alpha=70^{\circ}, 90^{\circ}, 110^{\circ}$. These were acquired without moving the probe.

Imaging was performed using an Acuson Sequoia Echo 512 machine with Siemens 6L3 probe. An ultrasound frequency of 3.5 MHz was used. The penetration depth is $30 \mathrm{~mm}$. Color calibration was done for the axial velocity ranging from $-0.17 \mathrm{~m} / \mathrm{s}$ to $0.17 \mathrm{~m} / \mathrm{s}$

We extracted the frames corresponding to the $R$-peak over two cycles. Fig. 7 gives one of the extracted frames for each view angle. The top of the color scale represents the peak negative axial velocity and the bottom represents the peak positive velocity. Note that the color map has been inverted for the first view when compared to the other two views. The noise variance of the Doppler data was estimated by comparing frames corresponding to different cycles. It was found to amount for $9 \%$ of the signal energy.

We used $75 \%$ of the total samples that were selected randomly (data set A) for reconstruction and the remaining samples (data set B) for validation. Since we do not have any $a$ priori knowledge about the flow pattern, all the four regularization parameters were tuned iteratively. The minimum attained [(51)] was in the order of $10^{-4}$. Fig. 8(a) gives a visualization of the reconstructed flow field, whereas the Fig. 8(b) gives the flow profiles across the vessels before and after the bifurcation. We observe that, in the flow profiles after bifurcation, the maximum velocity is shifted towards the interior of the bifurcation, whereas in the flow profile before bifurcation, the maximum velocity stays approximately in the center of the vessel. This 


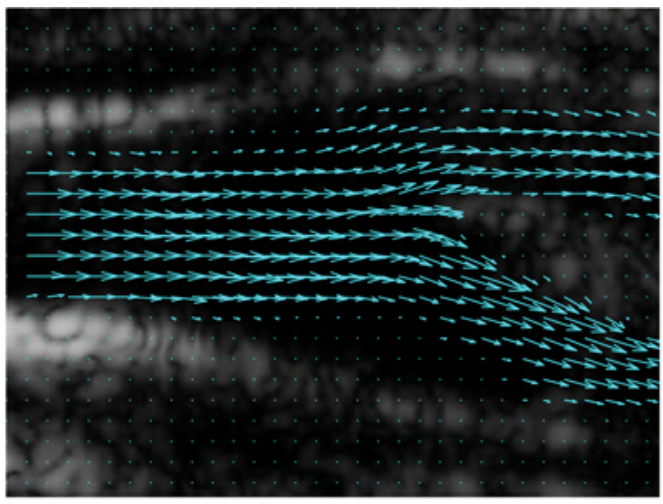

(a)

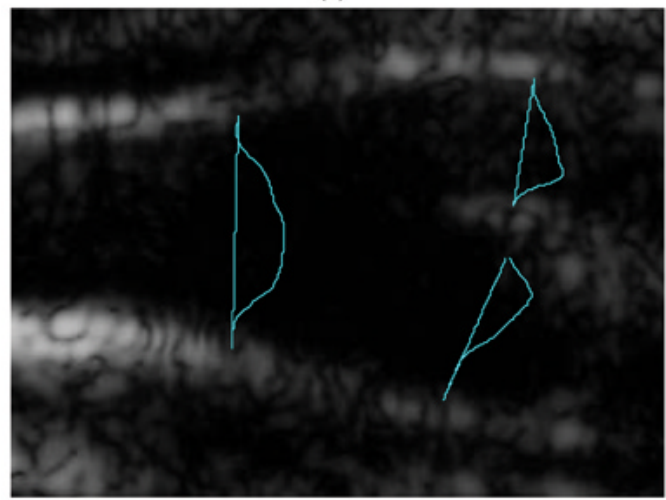

(b)

Fig. 8. Reconstructed results for the carotid bifurcation. (a) Reconstructed flow field. (b) Velocity profile across different parts of the vessels.

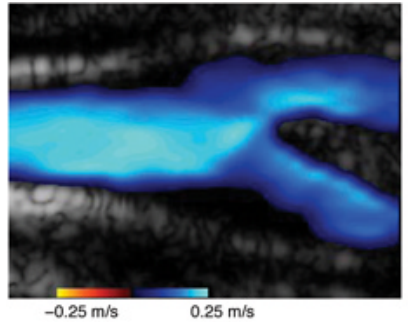

(a)

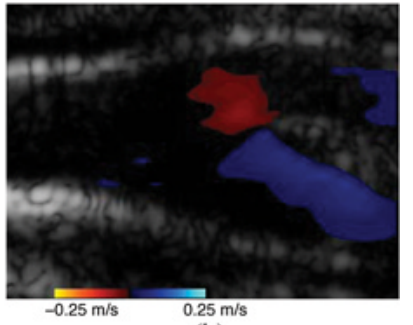

(b)
Fig. 9. Color coded reconstructed velocity components. (a) Horizontal component (left to right is positive). (b) Vertical component (downwards is positive).

agrees with model-based predictions [22], and constitutes a significant clinical finding. Fig. 9(a) and (b) visualizes the reconstructed velocity components in a color coded form.

Next, we cross validated the reconstructed flow field using data set B. Specifically, we evaluated the cost (51) for the reconstructed function. The cost was close to the attained minimum during the iterative search; i.e., of the order of $10^{-4}$. This indicates that the chosen values for the regularization parameters are matched to the data, and that the reconstructed velocity must be close to the underlying true flow field.

\section{G. Myocardial Motion Recovery}

The data for this experiment are from a healthy individual and were acquired using a sector scan probe from two echocardiographic standard views: 1) apical long axis view, and 2) parasternal long axis view.
Imaging was performed using a Philips SONOS 5500 system with S4 ultraband cardiac transducer. An ultrasound frequency of $1.8 \mathrm{MHz}$ was used. The penetration depth is $150 \mathrm{~mm}$. Color calibration was done for the axial velocity ranging from $-12 \mathrm{~m} / \mathrm{s}$ to $12 \mathrm{~m} / \mathrm{s}$.

Along with the Doppler images, the data set contains $B$-mode intensity images that reveal the anatomical structure of the cross sectional plane. The acquisition was done by an experienced echocardiographer who adjusted the probe such that both acquisitions are from the same cross-sectional plane. Hence, the premise of this data set is that the image planes are related by a rigid transformation (translation+rotation). To reconstruct the motion field in one image plane, one, therefore, has to register the two data sets and superimpose them onto a common reference system. To this end, we computed the required transformation by identifying a pair of landmarks (base of the mitral valves) in both the sequences.

The noise variance estimated using periodicity in this case is $22 \%$ of the signal energy. In this experiment too, we used $75 \%$ of the samples for reconstruction and the rest for validation. Parameter tuning was performed as in the previous experiment. The attained minimum of the cost (51) was in the order of $10^{-3}$ in this case and the cost for the validation data set was also of the same order.

Note that the minimum attained in this experiment is less than that of the previous experiment. This is probably due to the fact that there are two additional sources of error: 1) misalignment of cross-sectional planes, and 2) error in registering two images. The effect of the later can be reduced if one uses more landmarks.

By looking at the $B$-mode images, we manually generated regions of interest (binary masks) specifying the cardiac wall that is visible in the given images. Fig. 10 shows the reconstructed motion fields displayed over the regions of interest for the frame corresponding to the leading edge of the $T$-wave of the associated electrocardiogram (ECG). Note that, even though the reconstructed motion fields in the apical and parasternal planes are related by a transformation, the displayed motions fields do not look exactly so, since the regions of interest are slightly different.

The reconstructed velocity field was also validated in a qualitative manner by comparison of the motion directions determined from grayscale images by several echocardiographic experts. In this way, we could confirm that the results found by our algorithm are generally in good agreement with the expert readings.

\section{H. Discussion}

The above experimental examples demonstrate the effectiveness of the proposed method. We observe a striking difference between the performance of the vector regularizer and the more traditional thin-plate spline regularizer. This is due to the fact that the vector regularizer introduces a coupling between the $x$ and $y$ components whereas the thin-plate spline regularizer-which works well when the sample are complete (both components available) — treats the components independently. The incompleteness of the Doppler data makes the coupling crucial, and hence the improvement of coupled regularizer over 


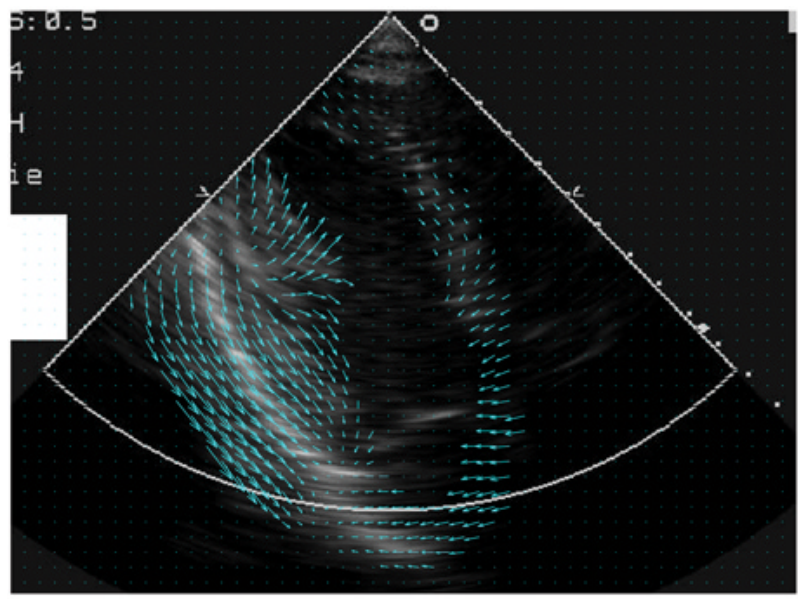

(a)

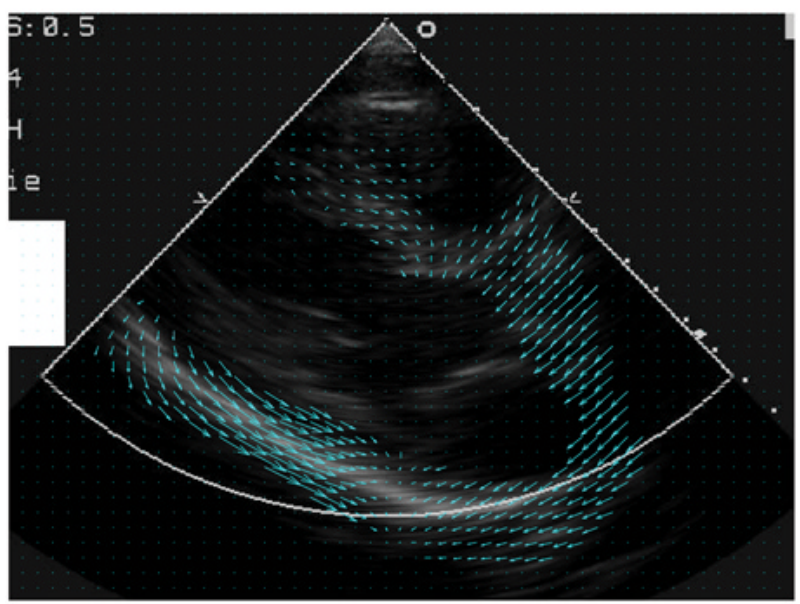

(b)

Fig. 10. Reconstructed motion field from two view cardiac Doppler data. (a) Apical long axis view. (b) Parasternal long axis view.

the uncoupled one is significant. Another point that makes the proposed vector regularizer attractive is that it allows one to incorporate some a priori knowledge on the motion. This term can be specified to penalize pure (or elastic) deformation only; in other words, the null space of the regularization operator can be chosen to include all forms of rigid motion such as translation and rotation.

Our reconstruction method is formulated in the continuous domain. Our choice of spline reconstruction space is computationally attractive; it allows an optimal trade-off between the complexity and accuracy, since $B$-splines have the maximum approximation order for a given support size [20] $\left(O\left(a^{n+1}\right)\right.$ convergence in the $L_{2}$ sense). Further, the exact solution is expressed simply in the form of a sparse linear system of equation. The proposed computational framework is general and flexible. The user can easily fine-tune the regularization for his particular application. Practically, changing the regularization functional only amounts to adjusting some weights or eventually adding a convolution matrix. In fact, one can use a set of templates of such matrices to get any quadratic functional as a weighted sum.

All the reconstructions were done with the grid size in the range 32-50. The corresponding size of the linear system is in the range $2048-5000$. Thanks to the right choice of the basis functions, the matrices are sparse and hence it takes only 3-9 s to solve by direct methods using Matlab on a Apple G5 system. We also implemented a multigrid algorithm [23] for the case $\lambda_{d_{0}}=$ $\lambda_{c_{0}}$, and $\lambda_{d_{1}}=\lambda_{c_{1}}$. We verified that the multigrid solver will speed up by at least one order of magnitude. The same strategy should also work for the more general case but will require a significant programming effort as well as a reengineering of the structure of the current program, which is an adaptation of the scalar algorithm developed in [24].

It should be noted that, as far as we know, our method is the first attempt to recover vector velocity field from Doppler data only. Our method opens up new possibilities in clinical diagnosis. In particular, we can now recover full velocity field from blood flow, which has not been possible before. Further, for tissue motion, our method yields more accurate result compared to intensity-based approaches due to the reasons explained in the introduction. We believe that integrating our algorithm in imaging machines that have capability of obtaining multiple view acquisitions will be a promising direction, since this will eliminate the inaccuracies due to manual alignment.

Another strength of the proposed method is that it can integrate multiple acquisitions efficiently. This is due fact that 1) the method is formulated for arbitrarily spaced samples and 2) number of sample points does not significantly affect the computational complexity.

Finally, it should be noted that the proposed formulation is perfectly extendable to thee-dimensional (3-D). The only practical difficulty there is to acquire synchronized multiple view data. Fortunately, the prospects are getting better with the recent generation of 3-D probes.

\section{Conclusion}

We demonstrated the feasibility of recovering true velocity field from pulsed wave Doppler data. We adopted a minimization approach, where the cost to be minimized is a weighted sum of data error and a smoothness functional. We proposed a derivative-based smoothness functional that allows one to incorporate an a priori knowledge about the motion and set up to penalize the deformation. We proposed a continuous solution for this minimization problem, and showed how the solution could be obtained by solving a system of linear equations. We demonstrated the effectiveness of the method using experimental examples. We also demonstrated the importance of tuning the smoothness functional, and showed how the data indeterminacy can be overcome by a suitable choice of vector regularization.

\section{APPENDIX}

Our goal is to find expressions for $R(\mathbf{v})$ and $R_{E}(\mathbf{v})$ in terms of the $B$-spline expansion coefficients. Each constitutive term is a bilinear functional involving the velocity components. They are of the following form:

$$
\begin{aligned}
& B\left(f, g, p_{1}, q_{1}, p_{2}, q_{2}\right) \\
& \quad=\int\left(\partial_{x}^{p_{1}} \partial_{y}^{q_{1}} f(x, y)\right)\left(\partial_{x}^{p_{2}} \partial_{y}^{q_{2}} g(x, y)\right) d x d y
\end{aligned}
$$


where $f$ and $g$ are expressed as

$$
\begin{aligned}
& f(x, y)=\sum_{k, l} c_{k, l}^{f} \beta^{n}\left(\frac{x}{a}-k\right) \beta^{n}\left(\frac{y}{a}-l\right) \\
& g(x, y)=\sum_{m, n} c_{m, n}^{g} \beta^{n}\left(\frac{x}{a}-m\right) \beta^{n}\left(\frac{y}{a}-n\right) .
\end{aligned}
$$

Here, $f$ and $g$ are the symbolic replacements for the velocity components, $u$ and $v$; they either refer to the same component (square terms) or different components (cross terms). This representation facilitates deriving a generic computational formula that will be useful for both square terms and cross terms. To derive the formula, we first define

$$
\alpha_{p, a}(x)=\frac{d^{p}}{d x^{p}}\left\{\beta^{n}\left(\frac{x}{a}\right)\right\} .
$$

Substituting (54) and (55) in (53), we get the equation at the bottom of the page. This in turn yields

$$
\begin{aligned}
& B\left(f, g, p_{1}, q_{1}, p_{2}, q_{2}\right) \\
& \quad=\sum_{l, n} \sum_{k, m} c_{k, l}^{f} c_{m, n}^{g} \eta_{p_{1}, p_{2}}((m-k) a) \eta_{q_{1}, q_{2}}((n-l) a)
\end{aligned}
$$

where $\eta_{p_{1}, p_{2}}(x)$ is defined by the following convolution:

$$
\eta_{p_{1}, p_{2}}(x)=\alpha_{p_{1}, a}(x) * \alpha_{p_{2}, a}(-x) .
$$

Now define

$$
\begin{aligned}
\gamma_{p_{1}, p_{2}}(k) & =\eta_{p_{1}, p_{2}}(k a) \\
\mu_{p_{1}, q_{1}, p_{2}, q_{2}}(k, l) & =\gamma_{p_{1}, p_{2}}(k) \gamma_{q_{1}, q_{2}}(l) .
\end{aligned}
$$

Substituting we get

$$
\begin{aligned}
B\left(f, g, p_{1}, q_{1}, p_{2}, q_{2}\right) & =\sum_{l, n} \sum_{k, m} c_{k, l}^{g} c_{m, n}^{f} \mu_{p_{1}, q_{1}, p_{2}, q_{2}}(m-k, n-l) \\
& =\sum_{k, l} c_{k, l}^{g} \sum_{m, n} c_{m, n}^{f} \mu_{p_{1}, q_{1}, p_{2}, q_{2}}(m-k, n-l) \\
& =\left\langle\mu_{p_{1}, q_{1}, p_{2}, q_{2}}(k, l) * c_{k, l}^{f}, \quad c_{k, l}^{g}\right\rangle .
\end{aligned}
$$

In other words, the bilinear form $B\left(f, g, p_{1}, q_{1}, p_{2}, q_{2}\right)$ that involves an integral over $\mathbb{R}^{2}$ is re-expressed as a bilinear form that involves a discrete inner product over $l_{2}$. Hence (61) gives a basic building block to represent any vector regularization functional for $B$-splines by discrete convolutions. The discrete filter $\mu_{p_{1}, q_{1}, p_{2}, q_{2}}(k, l)$ is completely determined by the quadruple of derivative orders $\left\{p_{1}, q_{1}, p_{2}, q_{2}\right\}$, the degree of the $B$-spline, and the scale $a$ [(56), (58), (59), (60)]. Let $\Gamma_{p_{1}, p_{2}}(z)$ and $M_{p_{1}, q_{1}, p_{2}, q_{2}}\left(z_{1}, z_{2}\right)$ be $z$-transforms of $\gamma_{p_{1}, p_{2}}(k)$ and $\mu_{p_{1}, q_{1}, p_{2}, q_{2}}(k, l)$, respectively. Then

$$
M_{p_{1}, q_{1}, p_{2}, q_{2}}\left(z_{1}, z_{2}\right)=\Gamma_{p_{1}, p_{2}}\left(z_{1}\right) \Gamma_{q_{1}, q_{2}}\left(z_{2}\right) .
$$

The next step now is to find explicit expression for $\Gamma_{p_{1}, p_{2}}(z)$. To this end, we first write the Fourier expressions for $B$-spline [16]:

$$
\mathcal{F}\left[\beta^{n}(x)\right]=\hat{\beta}^{n}(\omega)=\frac{\left(e^{j \omega / 2}-e^{-j \omega / 2}\right)^{n+1}}{(j \omega)^{n+1}} .
$$

Let $\beta_{a}^{n}(x)=\beta^{n}(x / a)$. Then

$$
\mathcal{F}\left[\beta_{a}^{n}(x)\right]=\hat{\beta}_{a}^{n}(\omega)=a \hat{\beta}^{n}(a \omega)=a \frac{\left(e^{j a \omega / 2}-e^{-j a \omega / 2}\right)^{n+1}}{(j a \omega)^{n+1}} .
$$

Consequently, the Fourier expression for $\alpha_{p, a}(x)$ defined in the (56) is given by

$$
\begin{aligned}
\hat{\alpha}_{p, a}(\omega)= & (j \omega)^{p} \hat{\beta}_{a}^{n}(\omega) \\
= & (j \omega)^{p} a \hat{\beta}^{n}(a \omega) \\
= & (j \omega)^{p} a \frac{\left(e^{j a \omega / 2}-e^{-j a \omega / 2}\right)^{n+1}}{(j a \omega)^{n+1}} \\
= & \frac{1}{a^{p}}\left(e^{j a \omega / 2}-e^{-j a \omega / 2}\right)^{p} \\
& {\left[a \frac{\left(e^{j a \omega / 2}-e^{-j a \omega / 2}\right)^{n+1-p}}{(j a \omega)^{n+1-p}}\right] . }
\end{aligned}
$$

Now, $\eta_{p_{1}, p_{2}}(x)$ defined in the (58) is given by

$$
\hat{\eta}_{p_{1}, p_{2}}(\omega)=\hat{\alpha}_{p_{1}, a}(\omega) \tilde{\hat{\alpha}}_{p_{2}, a}(\omega) .
$$

Substituting (63) yields

$$
\begin{aligned}
\hat{\eta}_{p_{1}, p_{2}}(\omega)= & \frac{1}{a^{p_{1}+p_{2}}}\left(e^{j a \omega / 2}-e^{-j a \omega / 2}\right)^{p_{1}} \\
& \left(e^{-j a \omega / 2}-e^{j a \omega / 2}\right)^{p_{2}} \\
& a^{2}\left[\frac{\left(e^{j a \omega / 2}-e^{-j a \omega / 2}\right)^{n+1-p_{1}}}{(j a \omega)^{n+1-p_{1}}}\right] \\
& {\left[\frac{\left(e^{-j a \omega / 2}-e^{j a \omega / 2}\right)^{n+1-p_{2}}}{(-j a \omega)^{n+1-p_{2}}}\right] . }
\end{aligned}
$$

$$
B\left(f, g, p_{1}, q_{1}, p_{2}, q_{2}\right)=\sum_{l, n} \sum_{k, m} c_{k, l}^{f} c_{m, n}^{g} \int \alpha_{p_{1}, a}(x-k a) \alpha_{p_{2}, a}(x-m a) d x \int \alpha_{q_{1}, a}(y-l a) \alpha_{q_{2}, a}(y-n a) d y .
$$


Now let us consider the case when $p_{1}+p_{2}$ is even. We rewrite This yields the above equation as

$$
\begin{aligned}
\hat{\eta}_{p_{1}, p_{2}}(\omega)= & \frac{1}{a^{p_{1}+p_{2}-1}}\left(e^{j a \omega / 2}-e^{-j a \omega / 2}\right)^{p_{1}} \\
& \left(e^{-j a \omega / 2}-e^{j a \omega / 2}\right)^{p_{2}} \\
& a\left[\frac{\left(e^{j a \omega / 2}-e^{-j a \omega / 2}\right)^{2 n+2-p_{1}-p_{2}}}{(j a \omega)^{2 n+2-p_{1}-p_{2}}}\right] \\
= & \frac{1}{a^{p_{1}+p_{2}-1}}\left(e^{j a \omega / 2}-e^{-j a \omega / 2}\right)^{p_{1}} \\
& \left(e^{-j a \omega / 2}-e^{j a \omega / 2}\right)^{p_{2}} a \hat{\beta}^{2 n+1-p_{1}-p_{2}}(a \omega) \\
= & \frac{1}{a^{p_{1}+p_{2}-1}}\left(e^{j a \omega / 2}-e^{-j a \omega / 2}\right)^{p_{1}} \\
& \left(e^{-j a \omega / 2}-e^{j a \omega / 2}\right)^{p_{2}} \hat{\beta}_{a}^{2 n+1-p_{1}-p_{2}}(\omega) \\
= & \frac{1}{a^{p_{1}+p_{2}-1}} \hat{\zeta}^{\left(p_{1}, p_{2}\right)}\left(e^{j a \omega}\right) \hat{\beta}_{a}^{2 n+1-p_{1}-p_{2}}(\omega)(64)
\end{aligned}
$$

$$
\begin{aligned}
\gamma_{p_{1}, p_{2}}(k) & =\eta_{p_{1}, p_{2}}(k a) \\
& =\frac{1}{a^{p_{1}+p_{2}-1}} \sum_{l} \zeta_{l}^{\left(p_{1}, p_{2}\right)} \beta^{2 n+1-p_{1}-p_{2}}(k-l) \\
& =\frac{1}{a^{p_{1}+p_{2}-1}} \zeta_{k}^{\left(p_{1}, p_{2}\right)} * \beta^{2 n+1-p_{1}-p_{2}}(k) .
\end{aligned}
$$

Hence, in $z$-domain we get

$$
\Gamma_{p_{1}, p_{2}}(z)=\frac{1}{a^{p_{1}+p_{2}}-1} \hat{\zeta}^{\left(p_{1}, p_{2}\right)}(z) B^{2 n+1-p_{1}-p_{2}}(z)
$$

Now, let $p_{1}+p_{2}$ be odd. We rewrite (64) as follows:

$$
\begin{aligned}
& \hat{\eta}_{p_{1}, p_{2}}(\omega)=\frac{1}{a^{p_{1}+p_{2}-1}} \hat{\zeta}^{\left(p_{1}-1, p_{2}\right)}\left(e^{j a \omega}\right) \\
&\left(e^{j a \omega / 2}-e^{-j a \omega / 2}\right) \hat{\beta}_{a}^{2 n+1-p_{1}-p_{2}}(\omega) .
\end{aligned}
$$

$\hat{\zeta}^{\left(p_{1}, p_{2}\right)}\left(e^{j a \omega}\right)=\left(e^{j a \omega / 2}-e^{-j a \omega / 2}\right)^{p_{1}}\left(e^{-j a \omega / 2}-e^{j a \omega / 2}\right)^{p_{2}}$.

Note that $\hat{\zeta}^{\left(p_{1}-1, p_{2}\right)}\left(e^{j a \omega}\right)$ is now a polynomial in $e^{-j a \omega}$, and let

Since $p_{1}+p_{2}$ is even, $\hat{\zeta}^{\left(p_{1}, p_{2}\right)}\left(e^{j a \omega}\right)$ is a polynomial in $e^{-j a \omega}$ (or even polynomial in $e^{-j a \omega / 2}$ ). Let

$$
\hat{\zeta}^{\left(p_{1}, p_{2}\right)}\left(e^{j a \omega}\right)=\sum_{l} \zeta_{l}^{\left(p_{1}, p_{2}\right)} e^{-j l a \omega} .
$$

Substituting in (64) and applying an inverse Fourier transformation, we get

$$
\hat{\zeta}^{\left(p_{1}-1, p_{2}\right)}\left(e^{j a \omega}\right)=\sum_{l} \zeta_{l}^{\left(p_{1}-1, p_{2}\right)} e^{-j l a \omega} .
$$

Substituting the above equation in (66) and applying the inverse Fourier transformation, we get the first equation at the bottom of the page, This yields the second equation at the bottom of the page. where $d^{n}(x)=\beta^{n}(x+1 / 2)-\beta^{n}(x-1 / 2)$. In $z$-domain it reads

$$
\begin{aligned}
\eta_{p_{1}, p_{2}}(x) & =\frac{1}{a^{p_{1}+p_{2}-1}} \sum_{l} \zeta_{l}^{\left(p_{1}, p_{2}\right)} \beta_{a}^{2 n+1-p_{1}-p_{2}}(x-l a) \\
& =\frac{1}{a^{p_{1}+p_{2}-1}} \sum_{l} \zeta_{l}^{\left(p_{1}, p_{2}\right)} \beta^{2 n+1-p_{1}-p_{2}}\left(\frac{x}{a}-l\right) .
\end{aligned}
$$

$$
\begin{aligned}
\Gamma_{p_{1}, p_{2}}(z) & =\frac{1}{a^{p_{1}+p_{2}-1}} \hat{\zeta}^{\left(p_{1}-1, p_{2}\right)}(z) D^{2 n+1-p_{1}-p_{2}}(z), \\
\text { where } & \\
D^{n}(z) & =\sum_{k} d^{n}(k) z^{-k} .
\end{aligned}
$$

$$
\begin{aligned}
\eta_{p_{1}, p_{2}}(x) & =\frac{1}{a^{p_{1}+p_{2}-1}} \sum_{l} \zeta_{l}^{\left(p_{1}-1, p_{2}\right)}\left(\beta_{a}^{2 n+1-p_{1}-p_{2}}\left(x-l a+\frac{a}{2}\right)-\beta_{a}^{2 n+1-p_{1}-p_{2}}\left(x-l a-\frac{a}{2}\right)\right) \\
& =\frac{1}{a^{p_{1}+p_{2}-1}} \sum_{l} \zeta_{l}^{\left(p_{1}-1, p_{2}\right)}\left(\beta^{2 n+1-p_{1}-p_{2}}\left(\frac{x}{a}-l+\frac{1}{2}\right)-\beta^{2 n+1-p_{1}-p_{2}}\left(x-l-\frac{1}{2}\right)\right) .
\end{aligned}
$$

$$
\begin{aligned}
\gamma_{p_{1}, p_{2}}(k) & =\eta_{p_{1}, p_{2}}(k a) \\
& =\frac{1}{a^{p_{1}+p_{2}-1}} \sum_{l} \zeta_{l}^{\left(p_{1}-1, p_{2}\right)}\left(\beta^{2 n+1-p_{1}-p_{2}}\left(k-l+\frac{1}{2}\right)-\beta^{2 n+1-p_{1}-p_{2}}\left(k-l-\frac{1}{2}\right)\right) \\
& =\frac{1}{a^{p_{1}+p_{2}-1}} \zeta_{k}^{\left(p_{1}-1, p_{2}\right)} * d^{2 n+1-p_{1}-p_{2}}(k)
\end{aligned}
$$


In summary, the filter $\Gamma_{p_{1}, p_{2}}(z)$ is given by (67), shown at the bottom of the page, where

$$
\begin{aligned}
\hat{\zeta}^{\left(p_{1}, p_{2}\right)}(z) & =\left(z^{1 / 2}-z^{-1 / 2}\right)^{p_{1}}\left(z^{-1 / 2}-z^{1 / 2}\right)^{p_{2}} \\
B^{n}(z) & =\sum_{k} \beta^{n}(k) z^{-k} \\
D^{n}(z) & =\sum_{k}\left(\beta^{n}\left(k+\frac{1}{2}\right)-\beta^{n}\left(k-\frac{1}{2}\right)\right) z^{-k} .
\end{aligned}
$$

The filter, $\Gamma_{p_{1}, p_{2}}(z)$, has some symmetry properties. In (67), all the terms in the right hand side are symmetric except $D^{2 n+1-p_{1}-p_{2}}(z)$, which is anti-symmetric. Hence, we get

$$
\Gamma_{p_{1}, p_{2}}\left(z^{-1}\right)=(-1)^{p_{1}+p_{2}} \Gamma_{p_{1}, p_{2}}(z)
$$

On the other hand, (58) and (59) imply

$$
\Gamma_{p_{2}, p_{1}}(z)=\Gamma_{p_{1}, p_{2}}\left(z^{-1}\right) .
$$

This these two relations together yield

$$
\Gamma_{p_{2}, p_{1}}(z)=(-1)^{p_{1}+p_{2}} \Gamma_{p_{1}, p_{2}}(z)
$$

The next step is to use (67) to get explicit expressions for each of the terms in $R(\mathbf{v})$ and $R_{E}(\mathbf{v})$. We first consider $\mathcal{D}_{1}(u)$. Using (53), (15) is written as

$$
\mathcal{D}_{1}(u)=B(u, u, 1,0,1,0)+B(u, u, 0,1,0,1) .
$$

Substituting (61) in the above equation yields

$$
\begin{aligned}
\mathcal{D}_{1}(u)= & \left\langle\mu_{1,0,1,0}(k, l) * c_{k, l}^{u}, \quad c_{k, l}^{u}\right\rangle \\
& +\left\langle\mu_{0,1,0,1}(k, l) * c_{k, l}^{u}, \quad c_{k, l}^{u}\right\rangle \\
= & \left\langle\left(\mu_{1,0,1,0}(k, l)+\mu_{0,1,0,1}(k, l)\right) * c_{k, l}^{u}, \quad c_{k, l}^{u}\right\rangle .
\end{aligned}
$$

The constituent filters in the $z$-domain read

$$
\begin{aligned}
& M_{1,0,1,0}\left(z_{1}, z_{2}\right)=\Gamma_{1,1}\left(z_{1}\right) \Gamma_{0,0}\left(z_{2}\right) \\
& M_{0,1,0,1}\left(z_{1}, z_{2}\right)=\Gamma_{0,0}\left(z_{1}\right) \Gamma_{1,1}\left(z_{2}\right) .
\end{aligned}
$$

Using (67), the filters $\Gamma_{1,1}(z)$ and $\Gamma_{0,0}(z)$ are expressed as

$$
\begin{aligned}
& \Gamma_{0,0}(z)=B^{2 n+1}(z) \\
& \Gamma_{1,1}(z)=\left(z-2+z^{-1}\right) B^{2 n-1}(z) .
\end{aligned}
$$

Substituting these expressions in (71) yields (17). In a similar way, we can establish (18).

Next, we intend prove (28). We first expand $\int(\operatorname{Div} \mathbf{v}(\mathbf{x}))^{2} d \mathbf{x}$ using (25) and then substitute (53) for each resulting term. We get

$\int(\operatorname{Div} \mathbf{v}(\mathbf{x}))^{2} d \mathbf{x}$

$=B(u, u, 1,0,1,0)+B(v, v, 0,1,0,1)+2 B(u, v, 1,0,0,1)$.

Using (61), this yields

$$
\begin{gathered}
\int(\operatorname{Div} \mathbf{v}(\mathbf{x}))^{2} d \mathbf{x}= \\
\left\langle r_{11}(k, l) * c_{k, l}^{u}, \quad c_{k, l}^{u}\right\rangle \\
+\left\langle r_{12}(k, l) * c_{k, l}^{v}, \quad c_{k, l}^{v}\right\rangle \\
+\left\langle r_{13}(k, l) * c_{k, l}^{u}, \quad c_{k, l}^{v}\right\rangle \\
\text { where } \\
r_{11}(k, l)=\mu_{1,0,1,0}(k, l) \\
r_{12}(k, l)=\mu_{0,1,0,1}(k, l) \\
r_{13}(k, l)=\mu_{1,0,0,1}(k, l) .
\end{gathered}
$$$$
+\left\langle r_{12}(k, l) * c_{k, l}^{v}, \quad c_{k, l}^{v}\right\rangle
$$$$
+\left\langle r_{13}(k, l) * c_{k, l}^{u}, \quad c_{k, l}^{v}\right\rangle
$$

Using (62) and (67) results

$$
\begin{aligned}
R_{11}\left(z_{1}, z_{2}\right) & =\Gamma_{11}\left(z_{1}\right) \Gamma_{00}\left(z_{2}\right) \\
& =\left(z_{1}+2+z_{1}^{-1}\right) B^{2 n-1}\left(z_{1}\right) B^{2 n+1}\left(z_{2}\right) \\
R_{12}\left(z_{1}, z_{2}\right) & =\Gamma_{00}\left(z_{1}\right) \Gamma_{11}\left(z_{2}\right) \\
& =B^{2 n+1}\left(z_{1}\right)\left(z_{2}+2+z_{2}^{-1}\right) B^{2 n-1}\left(z_{2}\right) \\
R_{13}\left(z_{1}, z_{2}\right) & =\Gamma_{10}\left(z_{1}\right) \Gamma_{01}\left(z_{2}\right) \\
& =\Gamma_{10}\left(z_{1}\right) \Gamma_{10}\left(z_{2}^{-1}\right)(\text { using }(69)) \\
& =D^{2 n}\left(z_{1}\right) D^{2 n}\left(z_{2}^{-1}\right)
\end{aligned}
$$

which proves (28). We obtain (29) in a similar way.

Next, to establish (30), we first expand it as follows:

$$
\begin{aligned}
\int\|\nabla(\operatorname{Div} \mathbf{v}(\mathbf{x}))\|^{2} d \mathbf{x}= & B(u, u, 2,0,2,0) \\
& +B(v, v, 0,2,0,2) \\
& +B(u, u, 1,1,1,1) \\
& +B(v, v, 1,1,1,1) \\
& +B(u, v, 2,0,1,1) \\
& +B(u, v, 1,1,0,2) .
\end{aligned}
$$

Using (61) yields

$$
\begin{aligned}
\int\|\nabla(\operatorname{Div} \mathbf{v}(\mathbf{x}))\|^{2} d \mathbf{x}= & \left\langle r_{21}(k, l) * c_{k, l}^{u}, \quad c_{k, l}^{u}\right\rangle \\
& +\left\langle r_{22}(k, l) * c_{k, l}^{v}, \quad c_{k, l}^{v}\right\rangle \\
& +\left\langle r_{23}(k, l) * c_{k, l}^{u}, \quad c_{k, l}^{v}\right\rangle
\end{aligned}
$$

$$
\Gamma_{p_{1}, p_{2}}(z)= \begin{cases}\left(\frac{1}{a}\right)^{p_{1}+p_{2}-1} \hat{\zeta}^{\left(p_{1}, p_{2}\right)}(z) B^{2 n+1-p_{1}-p_{2}}(z), & \text { if } p_{1}+p_{2} \text { is even, and } \\ \left(\frac{1}{a}\right)^{p_{1}+p_{2}-1} \hat{\zeta}^{\left(p_{1}-1, p_{2}\right)}(z) D^{2 n+1-p_{1}-p_{2}}(z), & \text { otherwise }\end{cases}
$$


where

$$
\begin{aligned}
R_{21}\left(z_{1}, z_{2}\right) & =M_{2,0,2,0}\left(z_{1}, z_{2}\right)+M_{1,1,1,1}\left(z_{1}, z_{2}\right) \\
& =\Gamma_{22}\left(z_{1}\right) \Gamma_{00}\left(z_{2}\right)+\Gamma_{11}\left(z_{1}\right) \Gamma_{11}\left(z_{2}\right) \\
R_{22}\left(z_{1}, z_{2}\right) & =M_{0,2,0,2}\left(z_{1}, z_{2}\right)+M_{1,1,1,1}\left(z_{1}, z_{2}\right) \\
& =\Gamma_{00}\left(z_{1}\right) \Gamma_{22}\left(z_{2}\right)+\Gamma_{11}\left(z_{1}\right) \Gamma_{11}\left(z_{2}\right) \\
R_{23}\left(z_{1}, z_{2}\right) & =M_{2,0,1,1}\left(z_{1}, z_{2}\right)+M_{1,1,0,2}\left(z_{1}, z_{2}\right) \\
& =\Gamma_{21}\left(z_{1}\right) \Gamma_{01}\left(z_{2}\right)+\Gamma_{10}\left(z_{1}\right) \Gamma_{12}\left(z_{2}\right) .
\end{aligned}
$$

Now, using (70), we get $\Gamma_{21}\left(z_{1}\right) \Gamma_{01}\left(z_{2}\right)=$ $\left(-\Gamma_{12}\left(z_{1}\right)\right)\left(-\Gamma_{10}\left(z_{2}\right)\right)=\Gamma_{12}\left(z_{1}\right) \Gamma_{10}\left(z_{2}\right)$. Hence, $R_{23}\left(z_{1}, z_{2}\right)$ becomes

$$
R_{23}\left(z_{1}, z_{2}\right)=\Gamma_{12}\left(z_{1}\right) \Gamma_{10}\left(z_{2}\right)+\Gamma_{10}\left(z_{1}\right) \Gamma_{12}\left(z_{2}\right) .
$$

Applying the formula (67) in each of the terms in (72), (73), and (74), we finally get

$$
\begin{aligned}
R_{21}\left(z_{1}, z_{2}\right)= & \frac{1}{a^{2}}\left[\left(z_{1}-2+z_{1}^{-1}\right)^{2} B^{2 n-3}\left(z_{1}\right) B^{2 n+1}\left(z_{2}\right)\right. \\
& +\left(z_{1}-2+z_{1}^{-1}\right) B^{2 n-1}\left(z_{1}\right) \\
& \left.\left(z_{2}-2+z_{2}^{-1}\right) B^{2 n-1}\left(z_{2}\right)\right] \\
R_{22}\left(z_{1}, z_{2}\right)= & \frac{1}{a^{2}}\left[B^{2 n+1}\left(z_{1}\right)\left(z_{2}-2+z_{2}^{-1}\right)^{2} B^{2 n-3}\left(z_{2}\right)\right. \\
& +\left(z_{1}-2+z_{1}^{-1}\right) B^{2 n-1}\left(z_{1}\right) \\
& \left.\left(z_{2}-2+z_{2}^{-1}\right) B^{2 n-1}\left(z_{2}\right)\right] \\
R_{23}\left(z_{1}, z_{2}\right)= & \frac{1}{a^{2}}\left[\left(z_{1}-2+z_{1}^{-1}\right) D^{2 n-2}\left(z_{1}\right) D^{2 n}\left(z_{2}^{-1}\right)\right. \\
& \left.+D^{2 n}\left(z_{1}\right)\left(z_{2}-2+z_{2}^{-1}\right) D^{2 n-2}\left(z_{2}^{-1}\right)\right] .
\end{aligned}
$$

This proves (30). Equation (31) is established in a similar way.

\section{REFERENCES}

[1] D. Evans and W. McDicken, Doppler Ultrasound: Physics, Instrumentation and Signal Processing. New York: Wiley, 2000.

[2] G. Sutherland, M. Steward, K. Grounstroem, C. Moran, A. Fleming, F. Guell-Peris, R. Riemersma, L. Fenn, K. Fox, and W. Mc Dicken, "Color Doppler myocardial imaging: A new technique for assessment of myocardial function," J. Amer. Soc. Echocardiogr., vol. 7, no. 5, pp. 441-458, Sep.-Oct. 1994.

[3] P. Hunziker, M. Picard, N. Jander, M. Scherrer-Crosbie, M. Pfisterer, and P. Buser, "Regional wall motion assessment in stress echocardiography by tissue Doppler bull's-eyes," J. Amer. Soc. Echocardiogr., vol. 12, no. 3, pp. 196-202, Mar. 1999.

[4] H. Tsujino et al., "Combination of pulsed-wave Doppler and real-time three-dimensional color Doppler echocardiography for quantifying the stroke volume in the left ventricular outflow tract," Ultrasound Med. Biol., vol. 30, no. 11, pp. 1441-1446, 2004.
[5] C. Holland et al., "Volumetric flow estimation in vivo and in vitro using pulsed-Doppler ultrasound," Ultrasound Med. Biol., vol. 22, no. 5, pp. 591-603, 1996.

[6] C. DeGroot et al., "Evaluation of 3-D color Doppler ultrasound for the measurement of proximal isovelocity surface area," Ultrasound Med. Biol., vol. 26, no. 6, pp. 989-999, 2000.

[7] W. McDicken, G. Sutherland, C. Moran, and L. Gordon, "Colour Doppler velocity imaging of the myocardium," Ultrasound Med. Biol., vol. 18, no. 6-7, pp. 651-654, 1992.

[8] K. Miyatake, M. Yamagishi, N. Tanaka, M. Uematsu, N. Yamazaki, Y. Mine, A. Sano, and M. Hirama, "New method of evaluating left ventricular wall motion by color-coded tissue Doppler imaging: In vitro and in vivo studies," J. Amer. Coll. Cardiol., vol. 25, no. 3, pp. 717-724, Mar. 1995.

[9] J. Gorcsan, V. Gulati, W. Mandarino, and W. Katz, "Color-coded measures of myocardial velocity throughout the cardiac cycle by tissue Doppler imaging to quantify regional left ventricular function," Amer. Heart J., vol. 131, no. 6, pp. 1203-1213, Jun. 1996.

[10] L. Galiuto, G. Ignone, and A. DeMaria, "Contraction and relaxation velocities of the normal left ventricle using pulsed-wave tissue Doppler echocardiography," Amer. J. Cardiol., vol. 81, no. 5, pp. 609-614, Mar. 1998.

[11] P. Baraldi, A. Sarti, C. Lamberti, A. Prandini, and F. Sgallari, "Evaluation of differential optical flow techniques on synthesized echo images," IEEE Trans. Biomed. Eng., vol. 43, no. 3, pp. 259-272, Mar. 1996.

[12] M. Sühling, M. Arigovindan, C. Jansen, P. Hunziker, and M. Unser, "Myocardial motion analysis from B-mode echocardiograms," IEEE Trans. Image Process., vol. 14, no. 4, pp. 525-536, Apr. 2005.

[13] — - "Bimodal myocardial motion analysis from B-mode and tissue Doppler ultrasound," in Proc. 2nd 2004 IEEE Int. Symp. Biomed. Imag.: From Nano to Macro (ISBI'04), Arlington, VA, USA, Apr. 15-18, 2004, pp. 308-311.

[14] J. Meunier and M. Betrand, "Echocardiographic image mean gray levels changes with tissue dynamics," IEEE Trans. Biomed. Eng., vol. 42, no. 4, pp. 403-410, Apr. 1995.

[15] - "Ultrasonic texture motion analysis: Theory and simulation," IEEE Trans. Med. Imag., vol. 14, no. 2, pp. 293-300, Jun. 1995.

[16] M. Unser, "Splines: A perfect fit for signal and image processing," IEEE Signal Process. Mag., vol. 16, no. 2, pp. 22-38, Nov. 1999.

[17] J. Duchon, "Splines minimizing rotation-invariant semi-norms in sobolev spaces," Constructive Theory Functions Several Variables, pp. 85-100, 1977.

[18] F. L. Bookstein, Morphometric Tools for Landmark Data. Cambridge, U.K.: Cambridge Univ. Press, 1991.

[19] D. Suter and F. Chen, "Left ventricular motion reconstruction based on elastic vector splines," IEEE Trans. Med. Imag., vol. 19, no. 4, pp. 295-305, Apr. 2000

[20] T. Blu, P. Thevenaz, and M. Unser, "MOMS: Maximal-order interpolation of minimal support," IEEE Trans. Image Process., vol. 10, no. 7, pp. 1069-1080, Jul. 2001.

[21] D. Ku, D. Giddens, C. Zarins, and S. Glagov, "Pulsatile flow and artherosclerosis in the human carotid bifurcation-positive correlation between plaque location and oscillating shear stress," Artherosclerosis, pp. 293-302, 1985.

[22] D. Ku, "Blood flow in arteries," Аnпи. Rev. Fluid Mechanics, pp. 399-434, 1991.

[23] W. Hakbusch, Iterative Solutions of Large Sparse Systems of Equations. New York: Springer-Verlag, 1994.

[24] M. Arigovindan, M. Sühling, P. Hunziker, and M. Unser, "Variational image reconstruction from arbitrarily spaced samples: A fast multiresolution spline solution," IEEE Trans. Image Process., vol. 14, no. 4, pp. 450-460, Apr. 2005. 